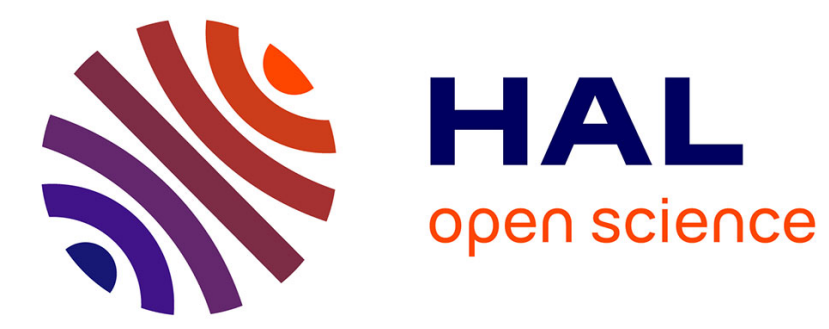

\title{
Traveling fronts for lattice neural field equations Grégory Faye
}

\section{- To cite this version:}

Grégory Faye. Traveling fronts for lattice neural field equations. Physica D: Nonlinear Phenomena, 2018, 378/379, pp.20-32. hal-01682252

\section{HAL Id: hal-01682252 https://hal.science/hal-01682252}

Submitted on 12 Jan 2018

HAL is a multi-disciplinary open access archive for the deposit and dissemination of scientific research documents, whether they are published or not. The documents may come from teaching and research institutions in France or abroad, or from public or private research centers.
L'archive ouverte pluridisciplinaire HAL, est destinée au dépôt et à la diffusion de documents scientifiques de niveau recherche, publiés ou non, émanant des établissements d'enseignement et de recherche français ou étrangers, des laboratoires publics ou privés. 


\title{
Traveling fronts for lattice neural field equations
}

\author{
Grégory Faye*1 \\ ${ }^{1}$ Institut de Mathématiques de Toulouse, UMR 5219, Université de Toulouse CNRS, UPS IMT, F-31062
}

Toulouse Cedex 9, France

October 5, 2017

\begin{abstract}
We show existence and uniqueness of traveling front solutions to a class of neural field equations set on a lattice with infinite range interactions in the regime where the kinetics of each individual neuron is of bistable type. The existence proof relies on a regularization of the traveling wave problem allowing us to use well-known existence results for traveling front solutions of continuous neural field equations. We then show that the traveling front solutions which have nonzero wave speed are unique (up to translation) by constructing appropriate sub and super solutions. The spectral properties of the traveling fronts are also investigated via a careful study of the linear operator around a traveling front in co-moving frame where we crucially use Fredholm properties of nonlocal differential operators previously obtained by the author in an earlier work. For the spectral analysis, we need to impose an extra exponential localization condition on the interactions.
\end{abstract}

\section{Introduction}

For $n \in \mathbb{Z}$, we consider the following lattice differential equation

$$
\dot{u}_{n}(t)=-u_{n}(t)+\sum_{j \in \mathbb{Z}} K_{j} S\left(u_{n-j}(t)\right), \quad t>0
$$

where $\dot{u}_{n}$ stands for $\frac{\mathrm{d} u_{n}}{\mathrm{~d} t}$ and $u_{n}(t)$ represents the membrane potential of neuron labelled $n$ at time $t$. Here $K_{j}$ represents the strength of interactions associated to the neural network at position $j$ on the lattice and the firing rate of neurons $S(u)$ is a nonlinear function. Such an equation can be seen as a Hopfield neural network model with infinite range interactions [19] or more simply as a discrete neural field equation [12] where each neuron is set on the lattice $\mathbb{Z}$ with all to all couplings.

\footnotetext{
*email: gregory.faye@math.univ-toulouse.fr
} 
In that later respect, we will call equation such as (1.1) Lattice Neural Field Equations (LNFEs). The continuous counter-part of $(1.1)$ is (see $[2,26])$

$$
\partial_{t} u(t, x)=-u(t, x)+\int_{\mathbb{R}} K(x-y) S(u(t, y)) \mathrm{d} y, \quad t>0, \quad x \in \mathbb{R} .
$$

Such a class of equations has received much attention in the past decades and has been very successful at reproducing a number of biological phenomena, including in particular visual hallucinations, binocular rivalry or working memory. We refer to the recent surveys $[7,8,11]$ for more developments on neural field models and applications in neuroscience. Equations such as (1.2) can also support a rich repertoire of phenomena, such as traveling waves, spatially periodic patterns, oscillatory dynamics and localized activity [8].

Our aim is to initiate a series of work on neural field dynamics set on various types of networks and equation (1.1) is one of the very first model to study as it consists of a network composed of infinite neurons indexed on $\mathbb{Z}$ with all to all couplings represented by the interaction communication rates $K_{j}$ for $j \in \mathbb{Z}$. There is a second natural motivation for studying LNFEs which stems from the numerical study of the continuous neural field equation (1.2). Indeed, if one is looking for a numerical approximation of the solutions of (1.2), one may discretize space and recover an equation similar to (1.1) depending on the quadrature rule used to approximate the integral in (1.2).

In the present paper, we would like to study special entire solutions of (1.1). Let first suppose that there exists two homogeneous stationary states $\left(u_{n}(t)\right)_{n \in \mathbb{Z}}=(u)_{n \in \mathbb{Z}}$ with $u=0$ and $u=1$ for the dynamics of (1.1). Hence, we are interested in particular solutions of (1.1) of the form $u_{n}(t)=\mathbf{u}(n-c t)$ for some $c \in \mathbb{R}$ where the profile $\mathbf{u}: \mathbb{R} \rightarrow \mathbb{R}$ satisfies

$$
\begin{aligned}
-c \mathbf{u}^{\prime}(x) & =-\mathbf{u}(x)+\sum_{j \in \mathbb{Z}} K_{j} S(\mathbf{u}(x-j)), \quad x \in \mathbb{R}, \\
\lim _{x \rightarrow-\infty} \mathbf{u}(x) & =1 \text { and } \lim _{x \rightarrow+\infty} \mathbf{u}(x)=0,
\end{aligned}
$$

where we set $x:=n-c t$ and $\mathbf{u}^{\prime}$ stands for $\frac{\mathrm{d} \mathbf{u}}{\mathrm{d} x}$. It is understood that when $c=0$, a stationary wave solution of (1.1) is a sequence $\left(u_{n}(t)\right)_{n \in \mathbb{Z}}=\left(\tilde{\mathbf{u}}_{n}\right)_{n \in \mathbb{Z}}$, independent of time, which verifies

$$
\begin{aligned}
\tilde{\mathbf{u}}_{n} & =\sum_{j \in \mathbb{Z}} K_{j} S\left(\tilde{\mathbf{u}}_{n-j}\right), \quad n \in \mathbb{Z}, \\
\lim _{n \rightarrow-\infty} \tilde{\mathbf{u}}_{n} & =1 \text { and } \lim _{n \rightarrow+\infty} \tilde{\mathbf{u}}_{n}=0 .
\end{aligned}
$$

The understanding of propagation phenomena in neural networks is crucial from a modeling point of view. Indeed, in the past few years, electrode recordings and imaging studies have revealed that the visual cortex can support a variety of cortical waves including standing waves, traveling pulses and spiral waves which are not only elicited by localized visual stimuli across the visual cortex but they are also present during spontaneous activity. We refer to [24] for recent review on the subject. It is thus important to understand the underlying mechanisms which allow the propagation of coherent structures such as traveling waves in neural network of the form of (1.1). 
The mathematical study of traveling waves in neural networks goes back to the pioneering work of Ermentrout and McLeod [13] for the continuous neural field equation (1.2) when the kinetics of the system is of bistable type. In [13], existence and uniqueness (up to translation) of monotone traveling front solutions were established. More recent works of the author deal with the existence of traveling fronts for monostable type of kinetics [14] and traveling pulses, i.e. non-monotone traveling waves connecting the same homogeneous stationary state, for continuous neural field models with a recovery variable, such as linear adaptation [17] or synaptic depression [15]. To the best of our knowledge, there are no available results regarding the existence, uniqueness and spectral stability of traveling front solutions for the LNFE (1.1) with infinite range interactions. If the support of the interactions were to be finite, then one could rely on the theory developed by Mallet-Paret [23] for the study of traveling front solutions in general lattice differential equations. Let us also mention the work of Bates and coauthors $[4,5]$ who studied traveling waves in infinite range lattice differential equations with bistable dynamics. The main difference between these works and our setting is that the nonlinearity appears within the infinite sum in (1.1) making the results of $[4,5]$ not readily available. Nevertheless, we will see that some of the ideas developed in [4] can be adapted to our setting.

Outline. The remainder of the paper is organized as follows. We give a precise statement of our assumptions and state our main results in Section 2. In Section 3, we prove the existence and uniqueness (up to translation) of monotone traveling front solutions of (1.1) (see Theorem 1 and 2). Section 4 is dedicated to the spectral properties of traveling front solutions with non zero wave speed (see Theorem 3). We conclude with a discussion in Section 5.

\section{Main results}

Throughout the paper, we will suppose that the following condition on the weights $K_{n}$ is satisfied

$$
\sum_{n \in \mathbb{Z}} K_{n}=1
$$

Then, steady homogeneous states of the form $\left(u_{n}(t)\right)_{n \in \mathbb{Z}}=(u)_{n \in \mathbb{Z}}$ for some $u \in \mathbb{R}$ satisfy the equation

$$
0=-u+S(u):=f(u) .
$$

We will assume the following hypotheses for the nonlinear function $S$.

Hypothesis (H1) - Bistable nonlinearity. We suppose that:

(i) $S \in \mathscr{C}_{b}^{r}(\mathbb{R})$ for $r \geq 2$ with $S(0)=0$ and $S(1)=1$ together with $S^{\prime}(0)<1$ and $S^{\prime}(1)<1$;

(ii) there exists a unique $\theta \in(0,1)$ such that $S(\theta)=\theta$ with $S^{\prime}(\theta)>1$; 
(iii) $u \mapsto S(u)$ is strictly nondecreasing on $[0,1]$ and there exists $s_{m}>1>s_{0}>0$ such that $s_{0}<S^{\prime}(u) \leq s_{m}$ for all $u \in[0,1]$.

The assumption (i)-(ii) ensures that $\left(u_{n}(t)\right)_{n \in \mathbb{Z}}=(u)_{n \in \mathbb{Z}}$ with $u \in\{0, \theta, 1\}$ are stationary homogeneous solutions of the LNFE (1.1) and that the function $f$ in (2.2) is of bistable type. The third condition ensures that $S$ is an increasing function, which is natural for a firing rate function. We also ask for some regularity for $S$, at least $\mathscr{C}_{b}^{2}(\mathbb{R})$. This will be necessary in order to prove our uniqueness result. Regarding the interaction weights $\left(K_{n}\right)_{n \in \mathbb{Z}}$, we will work with the following conditions.

Hypothesis (H2) - Weights. We suppose that:

(i) the normalization condition (2.1) is satisfied;

(ii) for all $n \in \mathbb{Z}$, we have $K_{n}=K_{-n} \geq 0$ and $K_{ \pm 1}>0$;

(iii) $\sum_{n \in \mathbb{Z}}|n| K_{n}<\infty$.

The second condition is a natural biological assumption and expresses the symmetric and excitatory nature of the considered neural network. The third condition is a technical assumption that is necessary in the process of proving the existence and uniqueness of traveling front solutions. Let us remark that our results cover both the case finite and infinite range interactions, although we are primarily interested in the later one where one may further assume that $K_{n}>0$ for all $n \in \mathbb{Z}$.

We are now in position to state the main results of the paper. The first result is about the existence of monotone traveling front solutions of (1.1).

Theorem 1 (Existence of monotone traveling waves). Suppose that the Hypotheses (H1)-(H2) are satisfied then there exists a traveling wave solution $u_{n}(t)=\mathbf{u}_{*}\left(n-c_{*} t\right)$ of (1.1) such that the profile $\mathbf{u}_{*}$ satisfies (1.3) when $c_{*} \neq 0$ or (1.4) if $c_{*}=0$. In the later case, we denote $\left(\tilde{\mathbf{u}}_{n}^{*}\right)_{n \in \mathbb{Z}}$ the stationary wave solution. Moreover,

(i) $\operatorname{sgn}\left(c_{*}\right)=\operatorname{sgn} \int_{0}^{1} f(u) \mathrm{d} u$ if $c_{*} \neq 0$;

(ii) if $\int_{0}^{1} f(u) \mathrm{d} u=0$ then $c_{*}=0$;

(iii) if $c_{*} \neq 0$ then $\mathbf{u}_{*} \in \mathscr{C}^{r+1}(\mathbb{R})$ and $\mathbf{u}_{*}^{\prime}<0$ on $\mathbb{R}$;

(iv) if $c_{*}=0$ then $\left(\tilde{\mathbf{u}}_{n}^{*}\right)_{n \in \mathbb{Z}}$ is a strictly decreasing sequence.

The proof of Theorem 1 relies on a strategy developed by Bates and Chmaj [4] for a discrete convolution model for phase transitions where the idea is to regularize the traveling wave problem (1.3). This amounts at considering a sequence of traveling waves problems for continuous neural field equations of the form of (1.2) and applying the results of Ermentrout \& McLeod [13]. The 
final step is to pass to the limit and verify that the limiting front profiles satisfy all the properties stated in Theorem 1.

The second result is about the uniqueness of traveling front solutions having nonzero wave speed.

Theorem 2 (Uniqueness of traveling waves with nonzero speed). Let $\left(\mathbf{u}_{*}, c_{*}\right)$ be a solution to (1.3) as given in Theorem 1, such that $c_{*} \neq 0$. Let $(\hat{\mathbf{u}}, \hat{c})$ be another solution to (1.3). Then $c=\hat{c}$ and, up to a translation, $\mathbf{u}_{*}=\hat{\mathbf{u}}$.

The strategy of proof of Theorem 2 is to use a "squeezing" technique developed by Chen in [10] by constructing appropriate sub and super solutions for (1.1). The principal difficulty here is that the nonlinearity enters in a non trivial way in the infinite sum, and thus we need to adapt all the arguments in our specific context. We rely on some comparison principles whose proofs are given in appendix.

Regarding the spectral stability of the traveling waves, we first require an extra assumption on the sequence of weights $\left(K_{j}\right)_{j \in \mathbb{Z}}$.

Hypothesis $(\mathbf{H} 2 \eta)$ - Exponential localization. We suppose that:

- $\left(K_{j}\right)_{j \in \mathbb{Z}}$ satisfies (H2);

- there exists $\eta>0$, such that $\sum_{j \in \mathbb{Z}} K_{j} e^{\eta|j|}<\infty$.

Our spectral result will be obtained for the continuous version of (1.1). That is we interpret solutions of $(1.1)$ as $u_{n}(t)=\mathbf{u}\left(t, n-c_{*} t\right)$ for some function $\mathbf{u} \in \mathscr{C}^{1}([0, \infty) \times \mathbb{R}, \mathbb{R})$, intuitively filing the gap between each lattice sites, which satisfies a nonlocal partial differential equation of the form

$$
\partial_{t} \mathbf{u}(t, x)=c_{*} \partial_{x} \mathbf{u}(t, x)-\mathbf{u}(t, x)+\int_{\mathbb{R}} K_{j} S(\mathbf{u}(t, x-j)), \quad(t, x) \in(0, \infty) \times \mathbb{R} .
$$

By definition, $\mathbf{u}_{*}$ from Theorems $1 \& 2$ is a stationary solution of the above equation and we will be interested by the spectral properties of its associated linear operator

$$
\mathcal{L} \mathbf{v}:=c_{*} \mathbf{v}^{\prime}-\mathbf{v}+\mathcal{K}_{\delta} *\left[S^{\prime}\left(\mathbf{u}_{*}\right) \mathbf{v}\right],
$$

where $\mathcal{K}_{\delta} * \mathbf{v}:=\sum_{j \in \mathbb{Z}} K_{j} \mathbf{v}(\cdot-j)$. From its definition, the operator $\mathcal{L}$ is a closed unbounded operator on $L^{2}(\mathbb{R})$ with dense domain $H^{1}(\mathbb{R})$ in $L^{2}(\mathbb{R})$. Furthermore, it is not difficult to check that $\mathcal{L}$ is the infinitesimal generator of a strongly continuous semigroup on $L^{2}(\mathbb{R})$ (see Lemma 4.1). Our main result regarding $\mathcal{L}$ reads as follows.

Theorem 3 (Spectral properties of $\mathcal{L})$. Suppose that the Hypotheses (H1)-(H2 $\eta$ ) are satisfied and let $\left(\mathbf{u}_{*}, c_{*}\right)$, with $c_{*} \neq 0$, be the unique (up to translation) strictly monotone traveling wave solution to (1.1) as given in Theorem 1. Let $\mathcal{L}: H^{1}(\mathbb{R}) \rightarrow L^{2}(\mathbb{R})$ be the operator defined in (2.3). We have:

(i) 0 is an algebraically simple eigenvalue of $\mathcal{L}$ with a negative eigenfunction $\mathbf{u}_{*}^{\prime}$; 
(ii) the adjoint operator $\mathcal{L}^{*}$ has a negative eigenfunction, denoted $\mathbf{q} \in \mathscr{C}^{1}(\mathbb{R})$, corresponding to the simple eigenvalue 0 ;

(iii) for all $0<\kappa<\min \left\{1-S^{\prime}(0), 1-S^{\prime}(1)\right\}$ the operator $\mathcal{L}-\lambda$ is invertible as an operator from $H^{1}(\mathbb{R})$ to $L^{2}(\mathbb{R})$ for all $\lambda \in \mathbb{C} \backslash 2 \pi \mathbf{i} c_{*} \mathbb{Z}$ such that $\Re(\lambda) \geq-\kappa ;$

(iv) there exist $\eta_{*}, \eta_{* *} \in(0, \eta)$ and some constants $C_{*}>0, C_{* *}>0$ such that

$$
\left|\mathbf{u}_{*}^{\prime}(x)\right| \leq C_{*} e^{-\eta_{*}|x|}\|\mathbf{u}\|_{L^{\infty}(\mathbb{R})}, \text { and }|\mathbf{q}(x)| \leq C_{*} e^{-\eta_{*}|x|}\|\mathbf{q}\|_{L^{\infty}(\mathbb{R})},
$$

for all $x \in \mathbb{R}$.

The main ingredient of the proof is to show that the operator $\mathcal{L}-\lambda$ is Fredholm on the some region in the complex plane. This analysis relies on some recent work [16] on Fredholm properties of nonlocal differential operators with infinite range interactions. Theorem 3 can be seen as preliminary result towards the nonlinear stability of traveling fronts of equation (1.1) (see the Discussion 5).

\section{Existence and uniqueness of monotone traveling waves}

In this section, we present the proofs of Theorems $1 \& 2$.

\subsection{Existence of monotone traveling waves - Proof of Theorem 1}

We follow the strategy developed by Bates \& Chmaj in [4] and define

$$
\mathcal{K}_{\delta}(x):=\sum_{j \in \mathbb{Z}} K_{j} \delta(x-j)
$$

where $\delta(x-j)$ stands for the delta Dirac mass at $x=j$. Using this notation, we can write

$$
\sum_{j \in \mathbb{Z}} K_{j} S(\mathbf{u}(x-j))=\mathcal{K}_{\delta} * S(\mathbf{u})[x]
$$

where $*$ denotes the convolution on the real line. As a consequence, the traveling wave problem (1.3) can be written as

$$
\begin{aligned}
-c \mathbf{u}^{\prime} & =-\mathbf{u}+\mathcal{K}_{\delta} * S(\mathbf{u}), \text { on } \mathbb{R}, \\
\lim _{x \rightarrow-\infty} \mathbf{u}(x) & =1 \text { and } \lim _{x \rightarrow+\infty} \mathbf{u}(x)=0 .
\end{aligned}
$$

Now, in order use the known results on the existence of monotone traveling waves of Ermentrout $\&$ McLeod [13], we need to regularize the kernel $\mathcal{K}_{\delta}$ in the following way. Let $\Psi \in \mathscr{C}^{\infty}(\mathbb{R}), \Psi \geq 0$, $\int_{\mathbb{R}} \Psi(x) \mathrm{d} x=1$, even and with compact support. Finally, define $\rho_{m}(x):=m \Psi(m x)$ for all $x \in \mathbb{R}$ and

$$
\mathcal{K}_{m}(x):=\sum_{j=-m}^{m} \frac{1}{\omega_{m}} K_{j} \rho_{m}(x-j),
$$


where $\omega_{m}:=\sum_{j=-m}^{m} K_{j}$. It then is easy to check (see [4]) that for all $\phi \in \mathscr{C}_{c}^{\infty}(\mathbb{R})$ we have

$$
\mathcal{K}_{m} * \phi \underset{m \rightarrow \infty}{\rightarrow} \mathcal{K}_{\delta} * \phi
$$

uniformly on compact sets. As a consequence, we can consider the sequence of traveling waves problems

$$
\begin{aligned}
-c_{m} \mathbf{u}_{m}^{\prime} & =-\mathbf{u}_{m}+\mathcal{K}_{m} * S\left(\mathbf{u}_{m}\right), \text { on } \mathbb{R}, \\
\lim _{x \rightarrow-\infty} \mathbf{u}_{m}(x) & =1 \text { and } \lim _{x \rightarrow+\infty} \mathbf{u}_{m}(x)=0 .
\end{aligned}
$$

With the definition of $\mathcal{K}_{m}$ in (3.3) we can also easily check that all the conditions listed in [13] are satisfied:

- $\mathcal{K}_{m}$ is absolutely continuous, with $\mathcal{K}_{m}^{\prime} \in L^{1}(\mathbb{R})$;

- $\mathcal{K}_{m}$ is even, of mass one and positive;

for all $m \geq 0$. Then, there exists a unique solution (modulo translation) $\left(\mathbf{u}_{m}, c_{m}\right)$ of (3.4) which further satisfies $\mathbf{u}_{m}^{\prime}<0$ on $\mathbb{R}$. Moreover, we have that $c_{m}=0$ if and only if $\int_{0}^{1} f(u) \mathrm{d} u=0$ and otherwise $\operatorname{sgn}\left(c_{m}\right)=\operatorname{sgn} \int_{0}^{1} f(u) \mathrm{d} u$. The solutions $\left(\mathbf{u}_{m}, c_{m}\right)$ are of course weak solutions of (3.4), i.e. for any $\phi \in \mathscr{C}_{c}^{\infty}(\mathbb{R})$ they satisfy

$$
-c_{m} \int_{\mathbb{R}} \mathbf{u}_{m} \phi^{\prime} \mathrm{d} x+\int_{\mathbb{R}}\left(-\mathbf{u}_{m}+\mathcal{K}_{m} * S\left(\mathbf{u}_{m}\right)\right) \phi \mathrm{d} x=0 .
$$

Let suppose that $c_{m} \geq 0$ and take $\alpha \in(0, \theta)$ and translate each $\mathbf{u}_{m}$ so that $\mathbf{u}_{m}(0)=\alpha$. As $\left(\mathbf{u}_{m}\right)_{m \geq 0}$ is a sequence of strictly monotone functions, by Helly's theorem, we can extract a subsequence of $\mathbf{u}_{m}$, which we still denote by $\mathbf{u}_{m}$, converging pointwise to a monotone function $\mathbf{u}_{*}$ as $m \rightarrow \infty$. Note that by construction, we have $0 \leq \mathbf{u}_{m} \leq 1$ and thus $0 \leq \mathbf{u}_{*} \leq 1$. Lets us show that the sequence $\left(c_{m}\right)_{m \geq 0}$ is also uniformly bounded. Assume the contrary, that there is a sequence $c_{m} \rightarrow+\infty$ as $m \rightarrow \infty$. From (3.4) we have

$$
\left|-c_{m} \mathbf{u}_{m}^{\prime}(x)\right|=\left|-\mathbf{u}_{m}(x)+\mathcal{K}_{m} * S\left(\mathbf{u}_{m}\right)(x)\right| \leq 2, \quad \text { for all } x \in \mathbb{R} \text { and } m \geq 0,
$$

and thus $\left\|\mathbf{u}_{m}^{\prime}\right\|_{\infty} \rightarrow 0$ as $m \rightarrow \infty$. This implies that $\mathbf{u}_{*}$ is constant and thus $\mathbf{u}_{*}=\alpha$. This is a contradiction. Indeed, as $\alpha \in(0, \theta)$, we have $f(\alpha)=-\alpha+S(\alpha)<0$ but

$$
-c_{m} \mathbf{u}_{m}^{\prime}=-\mathbf{u}_{m}+\mathcal{K}_{m} * S\left(\mathbf{u}_{m}\right)=-\mathbf{u}_{m}+\mathcal{K}_{m} * \mathbf{u}_{m}+\mathcal{K}_{m} * f\left(\mathbf{u}_{m}\right) \geq 0 \text { on } \mathbb{R},
$$

and we deduce

$$
-\mathcal{K}_{m} * f\left(\mathbf{u}_{m}\right) \leq-\mathbf{u}_{m}+\mathcal{K}_{m} * \mathbf{u}_{m}
$$

that is

$$
0<-f(\alpha)=\lim _{m \rightarrow \infty}\left(-\mathcal{K}_{m} * f\left(\mathbf{u}_{m}\right)\right) \leq \lim _{m \rightarrow \infty}\left(-\mathbf{u}_{m}+\mathcal{K}_{m} * \mathbf{u}_{m}\right)=0
$$


Finally, by passing to another subsequence, we also have that $c_{m} \rightarrow c_{*}$, for some $c_{*} \geq 0$, as $m \rightarrow \infty$. We can now pass to the limit in (3.5), and we obtain that $\mathbf{u}_{*}$ is a weak solution of (3.2) as it satisfies

$$
-c_{*} \int_{\mathbb{R}} \mathbf{u}_{*} \phi^{\prime} \mathrm{d} x+\int_{\mathbb{R}}\left(-\mathbf{u}_{*}+\mathcal{K}_{\delta} * S\left(\mathbf{u}_{*}\right)\right) \phi \mathrm{d} x=0,
$$

for all $\phi \in \mathscr{C}_{c}^{\infty}(\mathbb{R})$. This follows from Lebesgue's dominated convergence theorem, the continuity of $S$ and the limit

$$
\int_{\mathbb{R}}\left(\mathcal{K}_{m} * S\left(\mathbf{u}_{m}\right)\right) \phi \mathrm{d} x=\int_{\mathbb{R}}\left(\mathcal{K}_{m} * \phi\right) S\left(\mathbf{u}_{m}\right) \mathrm{d} x \underset{m \rightarrow \infty}{\longrightarrow} \int_{\mathbb{R}}\left(\mathcal{K}_{\delta} * \phi\right) S\left(\mathbf{u}_{*}\right) \mathrm{d} x=\int_{\mathbb{R}}\left(\mathcal{K}_{\delta} * S\left(\mathbf{u}_{*}\right)\right) \phi \mathrm{d} x .
$$

As a consequence, when $c_{*} \neq 0$, the equality (3.6) implies that $\mathbf{u}_{*} \in W^{1, \infty}(\mathbb{R})$. A boostrap argument then show that $\mathbf{u}_{*} \in \mathscr{C}^{r+1}(\mathbb{R})$ and thus a traveling wave solution (1.1). If $c_{*}=0$, then

$$
\int_{\mathbb{R}}\left(-\mathbf{u}_{*}+\mathcal{K}_{\delta} * S\left(\mathbf{u}_{*}\right)\right) \phi \mathrm{d} x=0, \text { for all } \phi \in \mathscr{C}_{c}^{\infty}(\mathbb{R}),
$$

so that

$$
\mathbf{u}_{*}=\mathcal{K}_{\delta} * S\left(\mathbf{u}_{*}\right) \text { a.e. on } \mathbb{R} .
$$

Note that $\mathcal{K}_{\delta}$ is not a regularization kernel and thus $\mathbf{u}_{*}$ need not be continuous. However, $\mathbf{u}_{*}$ is monotone with $0 \leq \mathbf{u}_{*} \leq 1$, therefore it has only jump discontinuities and the set of these jump discontinuities is at most countable. Thus we can find a sequence $\left(\iota_{k}\right)_{k \geq 0}$ with $\iota_{k} \rightarrow 0$ as $k \rightarrow \infty$ such that $\mathbf{u}_{*}\left(n+\iota_{k}\right)$ is continuous at $n+\iota_{k}$ for all $n \in \mathbb{Z}$ and $k>0$. We get that

$$
\mathbf{u}_{*}\left(n+\iota_{k}\right)=\mathcal{K}_{\delta} * S\left(\mathbf{u}_{*}\right)\left(n+\iota_{k}\right)=\sum_{j \in \mathbb{Z}} K_{j} S\left(\mathbf{u}_{*}\left(n+\iota_{k}-j\right)\right)
$$

for all $n \in \mathbb{Z}$ and $k>0$. It follows that the sequence

$$
\tilde{\mathbf{u}}_{n}^{*}:=\lim _{k \rightarrow \infty} \mathbf{u}_{*}\left(n+\iota_{k}\right), \quad n \in \mathbb{Z},
$$

satisfies

$$
\tilde{\mathbf{u}}_{n}^{*}=\sum_{j \in \mathbb{Z}} K_{j} S\left(\tilde{\mathbf{u}}_{n-j}^{*}\right)
$$

so is a stationary solution of (1.1).

So far, we have shown the existence of a monotone traveling wave solution $\mathbf{u}_{*}\left(n-c_{*} t\right)$ of $(1.1)$, and it remains to show that

$$
\begin{aligned}
\lim _{x \rightarrow-\infty} \mathbf{u}_{*}(x) & =1 \quad \& \quad \lim _{x \rightarrow+\infty} \mathbf{u}_{*}(x)=0, \\
\mathbf{u}_{*}^{\prime} & <0 \text { on } \mathbb{R}
\end{aligned}
$$

when $c_{*} \neq 0$ and

$$
\lim _{n \rightarrow-\infty} \tilde{\mathbf{u}}_{n}^{*}=1 \quad \& \quad \lim _{n \rightarrow+\infty} \tilde{\mathbf{u}}_{n}^{*}=0,
$$

$\left(\tilde{\mathbf{u}}_{n}^{*}\right)_{n \in \mathbb{Z}}$ is strictly decreasing ,

when $c_{*}=0$. 
Case $c_{*} \neq 0$. Without loss of generality, let assume that $c_{*}>0$, the argument for $c_{*}<0$ being the same. First, let us suppose that there exists $x_{0} \in \mathbb{R}$ such that $\mathbf{u}_{*}^{\prime}\left(x_{0}\right)=0$, with $\mathbf{u}_{*}^{\prime}(x) \leq 0$, we must have $\mathbf{u}_{*}^{\prime \prime}\left(x_{0}\right)=0$. Differentiating

$$
-c_{*} \mathbf{u}_{*}^{\prime}=-\mathbf{u}_{*}+\mathcal{K}_{\delta} * S\left(\mathbf{u}_{*}\right),
$$

and evaluating at $x=x_{0}$ we get

$$
0=-c_{*} \mathbf{u}_{*}^{\prime \prime}\left(x_{0}\right)=-\mathbf{u}_{*}^{\prime}\left(x_{0}\right)+\mathcal{K}_{\delta} *\left(\mathbf{u}_{*}^{\prime} S^{\prime}\left(\mathbf{u}_{*}\right)\right)\left[x_{0}\right]=\sum_{j \in \mathbb{Z}} K_{j} \mathbf{u}_{*}^{\prime}\left(x_{0}-j\right) S^{\prime}\left(\mathbf{u}_{*}\left(x_{0}-j\right)\right) .
$$

From the strict monotonicity of $S$, we get $S^{\prime}\left(\mathbf{u}_{*}\left(x_{0}-j\right)\right)>0$ for all $j \in \mathbb{Z}$ and thus $\mathbf{u}_{*}^{\prime}\left(x_{0}-j\right)=0$ for all $j$ such that $K_{j}>0$. Since, we assumed that $K_{ \pm 1}>0$ we deduce that $\mathbf{u}_{*}^{\prime}\left(x_{0}+n\right)=0$ for all $n \in \mathbb{Z}$ by induction. We can now obtain a contradiction as in [4], by considering the following initial value problem:

$$
\begin{aligned}
\dot{w}_{n}(t) & =-w_{n}(t)+\sum_{j \in \mathbb{Z}} K_{j} S\left(w_{n-j}(t)\right), \quad t>0 \text { and } n \in \mathbb{Z}, \\
w_{n}\left(-x_{0} / c_{*}\right) & =\mathbf{u}_{*}\left(n+x_{0}\right) .
\end{aligned}
$$

We readily check that $\mathbf{u}_{*}\left(x-c_{*} t\right)$ is solution of (3.7) and since $\mathbf{u}_{*}^{\prime}\left(x_{0}+n\right)=0$ for all $n \in \mathbb{Z}$, the constant $w_{n}(t)=\mathbf{u}_{*}\left(n+x_{0}\right)$ also solves (3.7), contradicting the uniqueness of the solutions of the initial boundary value problem (3.7). As a consequence, we have $\mathbf{u}_{*}^{\prime}(x)<0$ for all $x \in \mathbb{R}$.

On the other hand, from the monotonicity of $\mathbf{u}_{*}$, we readily obtain that

$$
f\left(\mathbf{u}_{*}( \pm \infty)\right)=0,
$$

and as $\mathbf{u}_{*}(0)=\alpha \in(0, \theta)$ we necessarily have that $\mathbf{u}_{*}(+\infty)=0$ and $\mathbf{u}_{*}(-\infty) \in\{\theta, 1\}$. Let us suppose that $\mathbf{u}_{*}(-\infty)=\theta$, then for all $x \in \mathbb{R}$ we have, by strict monotonicity of $\mathbf{u}_{*}$,

$$
0<\mathbf{u}_{*}<\theta
$$

and thus $f\left(\mathbf{u}_{*}(x)\right)<0$ for all $x \in \mathbb{R}$. Finally, from the equality

$$
-c_{*} \mathbf{u}_{*}^{\prime}=-\mathbf{u}_{*}+\mathcal{K}_{\delta} * \mathbf{u}_{*}+\mathcal{K}_{\delta} * f\left(\mathbf{u}_{*}\right)
$$

we get for all $N \geq 0$

$$
c_{*} \int_{-N}^{N} \mathbf{u}_{*}^{\prime}(x) \mathrm{d} x+\int_{-N}^{N}\left(-\mathbf{u}_{*}+\mathcal{K}_{\delta} * \mathbf{u}_{*}\right)(x) \mathrm{d} x=-\int_{-N}^{N} \mathcal{K}_{\delta} * f\left(\mathbf{u}_{*}\right)(x) \mathrm{d} x>0 .
$$

As

$$
\int_{-N}^{N}\left(-\mathbf{u}_{*}+\mathcal{K}_{\delta} * \mathbf{u}_{*}\right)(x) \mathrm{d} x \longrightarrow 0
$$


as $N \rightarrow+\infty$, we get that $-c_{*} \theta \geq 0$ which is a contradiction. To obtain the limit (3.8), it is enough to remark that

$$
\begin{aligned}
\int_{-N}^{N}\left(-\mathbf{u}_{*}+K_{\delta} * \mathbf{u}_{*}\right)(x) \mathrm{d} x & =\int_{-N}^{N} \sum_{j \in \mathbb{Z}} K_{j}\left(\mathbf{u}_{*}(x-j)-\mathbf{u}_{*}(x)\right) \mathrm{d} x \\
& =-\sum_{j \in \mathbb{Z}} j K_{j} \int_{-N}^{N}\left(\int_{0}^{1} \mathbf{u}_{*}^{\prime}(x-j \tau) \mathrm{d} \tau\right) \mathrm{d} x \\
& =-\sum_{j \in \mathbb{Z}} j K_{j} \int_{0}^{1}\left(\mathbf{u}_{*}(N-j \tau)-\mathbf{u}_{*}(-N-j \tau)\right) \mathrm{d} \tau \\
& \underset{N \rightarrow \infty}{\longrightarrow} \theta \sum_{j \in \mathbb{Z}} j K_{j}=0 .
\end{aligned}
$$

where we have used Lebesgue's dominated convergence theorem, Fubini's theorem, and [4, Lemma 2.1], together with the facts that $\sum_{j \in \mathbb{Z}}|j| K_{j}<\infty$ and $K_{j}=K_{-j}$ for all $j \in \mathbb{Z}$.

Case $c_{*}=0$. Let us first suppose that there exists $n_{0} \in \mathbb{Z}$ such that $\tilde{\mathbf{u}}_{n_{0}+1}^{*}=\tilde{\mathbf{u}}_{n_{0}}^{*}$, where the sequence $\tilde{\mathbf{u}}_{n}^{*}$ is solution of

$$
\tilde{\mathbf{u}}_{n}^{*}=\sum_{j \in \mathbb{Z}} K_{j} S\left(\tilde{\mathbf{u}}_{n-j}^{*}\right)
$$

for all $n \in \mathbb{Z}$. Thus, we have

$$
\sum_{j \in \mathbb{Z}} K_{j}\left(S\left(\tilde{\mathbf{u}}_{n_{0}+1-j}^{*}\right)-S\left(\tilde{\mathbf{u}}_{n_{0}-j}\right)^{*}\right)=0
$$

and then

$$
S\left(\tilde{\mathbf{u}}_{n_{0}+1-j}^{*}\right)=S\left(\tilde{\mathbf{u}}_{n_{0}-j}^{*}\right)
$$

for any $j \in \mathbb{Z}$ where $K_{j}>0$. As $S$ is strictly monotone and as we supposed that $K_{ \pm 1}>0$, by induction, it then follows that $\tilde{\mathbf{u}}_{n}^{*}$ is constant, a contradiction.

As in the case $c_{*} \neq 0$, we have that

$$
f\left(\lim _{n \rightarrow \pm \infty} \tilde{\mathbf{u}}_{n}^{*}\right)=0
$$

and thus $\lim _{n \rightarrow+\infty} \tilde{\mathbf{u}}_{n}^{*}=0$ and $\lim _{n \rightarrow-\infty} \tilde{\mathbf{u}}_{n}^{*} \in\{\theta, 1\}$. Let us suppose that $\lim _{n \rightarrow-\infty} \tilde{\mathbf{u}}_{n}^{*}=\theta$, then by strict monotonicity of $\tilde{\mathbf{u}}_{n}^{*}$ we have $0 \stackrel{n \rightarrow-\infty}{<} \tilde{\mathbf{u}}_{n}^{*}<\theta$ for all $n \in \mathbb{Z}$ and

$$
-\tilde{\mathbf{u}}_{n}^{*}+\sum_{j \in \mathbb{Z}} K_{j} \tilde{\mathbf{u}}_{n-j}^{*}=-\sum_{j \in \mathbb{Z}} K_{j} f\left(\tilde{\mathbf{u}}_{n-j}^{*}\right)>0 .
$$

But as for any $j \in \mathbb{Z}$ and all $N \geq 1$ we have that, by monotonicity of the sequence $\tilde{\mathbf{u}}_{n}^{*}$,

$$
\left|\sum_{n=-N}^{N}\left(\tilde{\mathbf{u}}_{n-j}^{*}-\tilde{\mathbf{u}}_{n}^{*}\right)\right| \leq 2 \theta|j| \text { and } \lim _{N \rightarrow \infty} \sum_{n=-N}^{N}\left(\tilde{\mathbf{u}}_{n-j}^{*}-\tilde{\mathbf{u}}_{n}^{*}\right)=j \theta,
$$


we have that

$$
\begin{aligned}
\sum_{n=-N}^{N}\left(-\tilde{\mathbf{u}}_{n}^{*}+\sum_{j \in \mathbb{Z}} K_{j} \tilde{\mathbf{u}}_{n-j}^{*}\right) & =\sum_{j} K_{j}\left(\sum_{n=-N}^{N}\left(\tilde{\mathbf{u}}_{n-j}^{*}-\tilde{\mathbf{u}}_{n}^{*}\right)\right), \\
& \underset{N \rightarrow \infty}{\longrightarrow} \theta \sum_{j \in \mathbb{Z}} j K_{j}=0
\end{aligned}
$$

we readily obtain a contradiction.

Proof of statements (i)-(ii) of Theorem 1. Suppose that $c_{*} \neq 0$. Then multiply (3.2a) by $S^{\prime}\left(\mathbf{u}_{*}\right) \mathbf{u}_{*}^{\prime}$ as in [13] to obtain

$$
-c_{*}\left(\mathbf{u}_{*}^{\prime}\right)^{2} S^{\prime}\left(\mathbf{u}_{*}\right)=\underbrace{\left(-\mathbf{u}_{*}+S\left(\mathbf{u}_{*}\right)\right) S^{\prime}\left(\mathbf{u}_{*}\right) \mathbf{u}_{*}^{\prime}}_{:=\mathcal{I}_{1}}+\underbrace{\left(\mathcal{K}_{\delta} * S\left(\mathbf{u}_{*}\right)\right) S^{\prime}\left(\mathbf{u}_{*}\right) \mathbf{u}_{*}^{\prime}-S\left(\mathbf{u}_{*}\right) S^{\prime}\left(\mathbf{u}_{*}\right) \mathbf{u}_{*}^{\prime}}_{:=\mathcal{I}_{2}} .
$$

Note that the last two terms of the previous equation can be written

$$
\mathcal{I}_{2}(x)=\int_{\mathbb{R}} \mathcal{K}_{\delta}(x-y)\left(S\left(\mathbf{u}_{*}(y)\right)-S\left(\mathbf{u}_{*}(x)\right)\right) S^{\prime}\left(\mathbf{u}_{*}(x)\right) \mathbf{u}_{*}^{\prime}(x) \mathrm{d} y
$$

and thus if we integrate over $\mathbb{R}$ we find

$\int_{\mathbb{R}} \mathcal{I}_{2}(x) \mathrm{d} x=\frac{1}{2} \int_{\mathbb{R}} \int_{\mathbb{R}} \mathcal{K}_{\delta}(x-y)\left(S\left(\mathbf{u}_{*}(y)\right)-S\left(\mathbf{u}_{*}(x)\right)\right)\left(S^{\prime}\left(\mathbf{u}_{*}(x)\right) \mathbf{u}_{*}^{\prime}(x)-S^{\prime}\left(\mathbf{u}_{*}(y)\right) \mathbf{u}_{*}^{\prime}(y)\right) \mathrm{d} y \mathrm{~d} x=0$.

As a consequence, we have that

$$
-c_{*} \int_{\mathbb{R}}\left(\mathbf{u}_{*}^{\prime}(x)\right)^{2} S^{\prime}\left(\mathbf{u}_{*}(x)\right) \mathrm{d} x=\int_{\mathbb{R}} \mathcal{I}_{1}(x) \mathrm{d} x=-\int_{0}^{1}(-u+S(u)) S^{\prime}(u) \mathrm{d} u .
$$

Finally, using the fact that $\int_{0}^{1}(-u+S(u))\left(S^{\prime}(u)-1\right) \mathrm{d} u=0$, we obtain that

$$
c_{*} \int_{\mathbb{R}}\left(\mathbf{u}_{*}^{\prime}(x)\right)^{2} S^{\prime}\left(\mathbf{u}_{*}(x)\right) \mathrm{d} x=\int_{0}^{1} f(u) \mathrm{d} u .
$$

It then follows that $\operatorname{sgn}\left(c_{*}\right)=\operatorname{sgn} \int_{0}^{1} f(u) \mathrm{d} u$ whenever $c_{*} \neq 0$ and that $\int_{0}^{1} f(u) \mathrm{d} u=0$ implies $c_{*}=0$. This concludes the proof of Theorem 1 .

\subsection{Uniqueness of traveling waves with nonzero speed - Proof of Theorem 2}

Throughout this sequel, we suppose that $\left(\mathbf{u}_{*}, c_{*}\right)$ is the strictly monotone traveling wave solution given by Theorem 1 and without loss of generality we may assume $c_{*}>0$. Let $(\hat{\mathbf{u}}, \hat{c})$ be another solution of (1.3). Before starting the proof of Theorem 2, we make the following remark. By construction, we know that $\mathbf{u}_{*} \in(0,1)$ and and it is also true that

$$
0 \leq \hat{\mathbf{u}} \leq 1, \quad \text { on } \mathbb{R}
$$


Suppose first that $\hat{c} \neq 0$, the case $\hat{c}=0$ can be handled in a similar way. By contradiction, if (3.9) were not satisfied, there would exist $x_{0} \in \mathbb{R}$ such that $\max _{x \in \mathbb{R}} \hat{\mathbf{u}}(x)=\hat{\mathbf{u}}\left(x_{0}\right)>1$. We obviously have $\hat{\mathbf{u}}^{\prime}\left(x_{0}\right)=0$ and $f\left(\hat{\mathbf{u}}\left(x_{0}\right)\right)<0$, and so

$$
0 \geq \mathcal{K}_{\delta} * S(\hat{\mathbf{u}})\left(x_{0}\right)-S\left(\hat{\mathbf{u}}\left(x_{0}\right)\right)=-f\left(\hat{\mathbf{u}}\left(x_{0}\right)\right)>0,
$$

gives a contradiction. Here we have used the monotonicity of $S$. Thus, we have $\hat{\mathbf{u}} \leq 1$ and a similar argument for the case where $\min _{x \in \mathbb{R}} \hat{\mathbf{u}}(x)=\hat{\mathbf{u}}\left(x_{0}\right)<0$ completes the proof of (3.9).

\subsubsection{Uniqueness of the wave speed}

Here, we are going to follow the strategy developed by Chen in [10], see also [1, 4]. The idea is to construct appropriate sub and super solutions for (1.1) based on the traveling wave solution $\mathbf{u}_{*}$ which will "squeeze" the other solution $\hat{\mathbf{u}}$. We also recall that a sub solution of (1.1) is a sequence $\left(u_{n}(t)\right)_{n \in \mathbb{Z}}$ which satisfies for all $n \in \mathbb{Z}$ and all $t>0$

$$
\dot{u}_{n}(t) \leq-u_{n}(t)+\sum_{j \in \mathbb{Z}} K_{j} S\left(u_{n-j}(t)\right) .
$$

A super solution is defined by reversing the inequality in (3.10).

We introduce two sequences

$$
w_{n}^{ \pm}(t):=\mathbf{u}_{*}\left(n-c_{*} t+\xi_{0} \mp \sigma \gamma\left(1-e^{-\beta t}\right)\right) \pm \gamma e^{-\beta t}, \quad \forall n \in \mathbb{Z}
$$

for some well chosen parameters $\xi_{0}, \sigma, \gamma$ and $\beta$. More precisely, we are going to prove the following key result.

Lemma 3.1. Assume that Hypotheses (H1)-(H2) hold and let $\left(\mathbf{u}_{*}, c_{*}\right)$ with $c_{*} \neq 0$ be as in Theorem (1). Then, there exists a small positive constant $\gamma_{0}$ and a large positive constant $\sigma$ such that for any $\gamma \in\left(0, \gamma_{0}\right]$ and every $\xi_{0} \in \mathbb{R}$, the sequences $w_{n}^{ \pm}(t)$ defined by (3.11) are a respectively sub $\left(w_{n}^{-}(t)\right)$ and super $\left(w_{n}^{+}(t)\right)$ solutions with $\beta:=\frac{1}{2} \min \left\{1-S^{\prime}(0) ; 1-S^{\prime}(1)\right\}>0$.

Proof. On only consider $w_{n}^{+}(t)$ as the proof for $w_{n}^{-}(t)$ is analogous. By assumption on the sequence $\left(K_{j}\right)_{j \in \mathbb{Z}}$, there exists $M_{0}>0$ such that

$$
\sum_{|j| \geq\left[M_{0}\right]} K_{j} \leq \frac{\beta}{8 s_{2}}
$$

where $\left[M_{0}\right]$ stands for nearest integer close to $M_{0}$. We define $\gamma_{0}>0$ by

$$
\gamma_{0}:=\min \left\{1, \frac{\beta}{8 s_{2}}\right\}
$$

where $s_{2}=\sup _{u \in[-1,2]}\left|S^{\prime \prime}(u)\right|$. Let $M_{1}>0$ be a constant such that

$$
\mathbf{u}_{*}(x)>1-\gamma_{0} \text { for all } x \leq-M_{1}, \quad \mathbf{u}_{*}(x)<\gamma_{0} \text { for all } x \geq M_{1} .
$$


We define

$$
\sigma:=\frac{\beta+s_{1}-1}{\min _{|x| \leq M_{0}+M_{1}}\left(-\beta \mathbf{u}_{*}^{\prime}(x)\right)}>0,
$$

where $s_{1}=\sup _{u \in[-1,2]}\left|S^{\prime}(u)\right|>s_{m}>1$ by Hypothesis (H1) on the nonlinearity. If we denote $\xi:=n-c_{*} t+\xi_{0}-\sigma \gamma\left(1-e^{-\beta t}\right)$, then we have

$$
\begin{aligned}
\mathbf{P}_{n}(t):=\dot{w}_{n}^{+}(t)+w_{n}^{+}(t)-\sum_{j \in \mathbb{Z}} K_{j} S\left(w_{n-j}^{+}(t)\right)= & \left(-c_{*}-\sigma \gamma \beta e^{-\beta t}\right) \mathbf{u}_{*}^{\prime}(\xi)-\gamma \beta e^{-\beta t}+\mathbf{u}_{*}(\xi)+\gamma e^{-\beta t} \\
& -\sum_{j \in \mathbb{Z}} K_{j} S\left(\mathbf{u}_{*}(\xi-j)+\gamma e^{-\beta t}\right) \\
= & -\sigma \gamma \beta e^{-\beta t} \mathbf{u}_{*}^{\prime}(\xi)-\gamma \beta e^{-\beta t}+\mathbf{u}_{*}(\xi)+\gamma e^{-\beta t} \\
& +\sum_{j \in \mathbb{Z}} K_{j}\left[S\left(\mathbf{u}_{*}(\xi-j)\right)-S\left(\mathbf{u}_{*}(\xi-j)+\gamma e^{-\beta t}\right)\right] .
\end{aligned}
$$

Let us then remark that

$$
\sum_{j \in \mathbb{Z}} K_{j}\left[S\left(\mathbf{u}_{*}(\xi-j)\right)-S\left(\mathbf{u}_{*}(\xi-j)+\gamma e^{-\beta t}\right)\right]=-\gamma e^{-\beta t} \sum_{j \in \mathbb{Z}} K_{j} \int_{0}^{1} S^{\prime}\left(\mathbf{u}_{*}(\xi-j)+\tau \gamma e^{-\beta t}\right) \mathrm{d} \tau
$$

with

$$
\left|\sum_{j \in \mathbb{Z}} K_{j} \int_{0}^{1} S^{\prime}\left(\mathbf{u}_{*}(\xi-j)+\tau \gamma e^{-\beta t}\right) \mathrm{d} \tau\right| \leq s_{1} .
$$

We can then write using the Lebesgue's dominated convergence theorem that

$$
\mathbf{P}_{n}(t)=\gamma e^{-\beta t}\left[-\beta \sigma \mathbf{u}_{*}^{\prime}(\xi)-\beta+1-\int_{0}^{1} \sum_{j \in \mathbb{Z}} K_{j} S^{\prime}\left(\mathbf{u}_{*}(\xi-j)+\tau \gamma e^{-\beta t}\right) \mathrm{d} \tau\right] .
$$

We are going to consider three separate regions:

$$
\text { (i) }|\xi| \leq M_{0}+M_{1}, \quad \text { (ii) } \xi>M_{0}+M_{1} \text {, and (iii) } \quad \xi<-M_{0}-M_{1} \text {. }
$$

Region (i). For $|\xi| \leq M_{0}+M_{1}$, we use the bound (3.13) to obtain

$$
\mathbf{P}_{n}(t) \geq \gamma e^{-\beta t}\left[-\beta \sigma \mathbf{u}_{*}^{\prime}(\xi)-\beta+1-s_{1}\right] \geq 0
$$

by definition of $\sigma$.

Region (ii). For $\xi>M_{0}+M_{1}$, we have $0<\mathbf{u}_{*}(\xi)<\gamma_{0}$. Furthermore, for all $\tau \in[0,1]$ we have that

$$
\begin{aligned}
\left|\sum_{j \in \mathbb{Z}} K_{j}\left[S^{\prime}\left(\mathbf{u}_{*}(\xi-j)+\tau \gamma e^{-\beta t}\right)-S^{\prime}(0)\right]\right| & \leq s_{2} \sum_{j \in \mathbb{Z}} K_{j}\left|\mathbf{u}_{*}(\xi-j)+\tau \gamma e^{-\beta t}\right| \\
& \leq 2 s_{2} \sum_{|j| \geq\left[M_{0}\right]} K_{j}+2 s_{2} \gamma_{0} \sum_{|j| \leq\left[M_{0}\right]-1} K_{j} \\
& \leq \frac{\beta}{2}
\end{aligned}
$$


As a consequence, we have that

$$
\begin{aligned}
\mathbf{P}_{n}(t) & =\gamma e^{-\beta t}\left[-\beta \sigma \mathbf{u}_{*}^{\prime}(\xi)-\beta+1-S^{\prime}(0)-\int_{0}^{1} \sum_{j \in \mathbb{Z}} K_{j}\left\{S^{\prime}\left(\mathbf{u}_{*}(\xi-j)+\tau \gamma e^{-\beta t}\right)-S^{\prime}(0)\right\} \mathrm{d} \tau\right] \\
& \geq \gamma e^{-\beta t}\left[-\beta \sigma \mathbf{u}_{*}^{\prime}(\xi)-\beta+1-S^{\prime}(0)-\frac{\beta}{2}\right] .
\end{aligned}
$$

Then, by definition of $\beta$ we have $-\beta+1-S^{\prime}(0)>\beta$ and thus $\mathbf{P}_{n}(t) \geq 0$ as $-\beta \sigma \mathbf{u}_{*}^{\prime}(\xi) \geq 0$.

Similarly, we can show that $\mathbf{P}_{n}(t) \geq 0$ in the region (iii). This completes the proof of the lemma.

We can now conclude the proof of Theorem 2 regarding the uniqueness of the wave speed.

Proof of Theorem 2 - Uniqueness of wave speed. Let us first suppose that $\hat{c} \neq 0$. We can then use Lemma 3.1 and a comparison principle (see Lemma A.1 in Appendix A) to squeeze $\hat{\mathbf{u}}$ between two translates of $\mathbf{u}_{*}$. First, since $\mathbf{u}_{*}$ and $\hat{\mathbf{u}}$ have the same limit at $\pm \infty$, there exists $h \gg 1$ such that

$$
\mathbf{u}_{*}(n)-\gamma_{0}<\hat{\mathbf{u}}(n)<\mathbf{u}_{*}(n-h)+\gamma_{0}, \quad \forall n \in \mathbb{Z} .
$$

We thus obtain for all $n \in \mathbb{Z}$ and all $t>0$ that

$$
\mathbf{u}_{*}\left(n-c_{*} t+\sigma \gamma\left(1-e^{-\beta t}\right)\right)-\gamma e^{-\beta t} \leq \hat{\mathbf{u}}(n-\hat{c} t) \leq \mathbf{u}_{*}\left(n-c_{*} t-h-\sigma \gamma\left(1-e^{-\beta t}\right)\right)+\gamma e^{-\beta t} .
$$

Then, we have in particular that

$$
\mathbf{u}_{*}\left(n-\hat{c} t+\left(\hat{c}-c_{*}\right) t+\sigma \gamma\left(1-e^{-\beta t}\right)\right)-\gamma e^{-\beta t} \leq \hat{\mathbf{u}}(n-\hat{c} t)
$$

and by letting $t \rightarrow+\infty$ while keeping $x=n-\hat{c} t$ fixed, we obtain that $\hat{c} \geq c_{*}$. Indeed assume otherwise, then one gets $1 \leq \hat{\mathbf{u}}(x)$ for all $x \in \mathbb{R}$ which is a contradiction. From the other inequality, we deduce that $\hat{c} \leq c_{*}$. As a conclusion, we have shown that $\hat{c}=c_{*}$. In addition, we also have that

$$
\mathbf{u}_{*}\left(x+\sigma \gamma_{0}\right) \leq \hat{\mathbf{u}}(x) \leq \mathbf{u}_{*}\left(x-h-\sigma \gamma_{0}\right), \quad x \in \mathbb{R} .
$$

When $\hat{c}=0$, we use a similar argument by first noting that there also exists $h \gg 1$ such that

$$
\mathbf{u}_{*}(n)-\gamma_{0}<\tilde{\mathbf{u}}_{n}^{*}<\mathbf{u}_{*}(n-h)+\gamma_{0}, \quad \forall n \in \mathbb{Z},
$$

and using Lemma 3.1 and a comparison principle, we get

$$
\mathbf{u}_{*}\left(n-c_{*} t+\sigma \gamma\left(1-e^{-\beta t}\right)\right)-\gamma e^{-\beta t} \leq \tilde{\mathbf{u}}_{n}^{*} \leq \mathbf{u}_{*}\left(n-c_{*} t-h-\sigma \gamma\left(1-e^{-\beta t}\right)\right)+\gamma e^{-\beta t} .
$$

Letting $t \rightarrow+\infty$ gives that $1 \leq \tilde{\mathbf{u}}_{n}^{*}$ for all $n \in \mathbb{Z}$, which is a contradiction, and thus $\hat{c} \neq 0$ which in turn implies $c_{*}=\hat{c}$ from the previous step. 


\subsubsection{Uniqueness of the profile up to translation}

To conclude the proof of Theorem 2, we need to show that $\mathbf{u}_{*}=\hat{\mathbf{u}}$, up to a translation. From (3.14), there exists a minimal $\bar{z}$ such that

$$
\mathbf{u}_{*}(x+z) \leq \hat{\mathbf{u}}(x) \text { for all } z>\bar{z} \text { and } x \in \mathbb{R}
$$

Let us suppose that $\mathbf{u}_{*}(\cdot+\bar{z}) \neq \hat{\mathbf{u}}$ and show that it leads to a contradiction. We proceed in two steps.

Step 1. If $\mathbf{u}_{*}(\cdot+\bar{z}) \neq \hat{\mathbf{u}}$ then necessarily $\mathbf{u}_{*}(x+\bar{z})<\hat{\mathbf{u}}(x)$ for all $x \in \mathbb{R}$. Otherwise, there exists some $x_{0} \in \mathbb{R}$ such that $\mathbf{u}_{*}\left(x_{0}+\bar{z}\right)=\hat{\mathbf{u}}\left(x_{0}\right)$. Let $\mathbf{w}(x):=\mathbf{u}_{*}(x+\bar{z})-\hat{\mathbf{u}}(x)$. Then, at $x=x_{0}$, we have $\mathbf{w}\left(x_{0}\right)=\mathbf{w}^{\prime}\left(x_{0}\right)=0$ and as both profiles $\hat{\mathbf{u}}$ and $\mathbf{u}_{*}$ satisfy the traveling wave equation (3.2a) for $c_{*}=\hat{c}$, we deduce that

$$
0=\mathcal{K}_{\delta} * S\left(\mathbf{u}_{*}\right)\left(x_{0}+\bar{z}\right)-\mathcal{K}_{\delta} * S(\hat{\mathbf{u}})\left(x_{0}\right)=\sum_{j \in \mathbb{Z}} K_{j}\left(S\left(\mathbf{u}_{*}\left(x_{0}-j+\bar{z}\right)\right)-S\left(\hat{\mathbf{u}}\left(x_{0}-j\right)\right)\right) \leq 0,
$$

and by positivity of $K_{j}$ and monotonicity of $S$ we have

$$
S\left(\mathbf{u}_{*}\left(x_{0}-j+\bar{z}\right)\right)-S\left(\hat{\mathbf{u}}\left(x_{0}-j\right)\right)=0,
$$

for all $j \in \mathbb{Z}$ where $K_{j}>0$. As by assumption $K_{ \pm 1}>0$, we have that $S\left(\mathbf{u}_{*}\left(x_{0}+n+\bar{z}\right)\right)=$ $S\left(\hat{\mathbf{u}}\left(x_{0}+n\right)\right)$ for all $n \in \mathbb{Z}$ and the strict monotonicity of $S$ implies that

$$
\mathbf{w}\left(x_{0}+n\right)=\mathbf{u}_{*}\left(x_{0}+n+\bar{z}\right)-\hat{\mathbf{u}}\left(x_{0}+n\right)=0, \quad n \in \mathbb{Z} .
$$

But, for all $n \in \mathbb{Z}$, the sequence of functions $w_{n}(t)=\mathbf{w}\left(x_{0}+n-c_{*} t\right)$ satisfies the following initial value problem

$$
\begin{aligned}
& \dot{w}_{n}(t)=-w_{n}(t)+\sum_{j \in \mathbb{Z}} K_{j} S^{\prime}\left(\zeta_{n-j}(t)\right) w_{n-j}(t), \quad t>0 \text { and } n \in \mathbb{Z}, \\
& w_{n}(0)=0
\end{aligned}
$$

for some $\zeta_{n-j}(t) \in\left(\mathbf{u}_{*}\left(x_{0}+n-j-c_{*} t+\bar{z}\right), \hat{\mathbf{u}}\left(x_{0}+n-j-c_{*} t\right)\right)$ which has a unique solution $w_{n}(t)=0$ and hence $\mathbf{w} \equiv 0$, a contradiction. As a consequence, we have $\mathbf{u}_{*}(x+\bar{z})<\hat{\mathbf{u}}(x)$ for all $x \in \mathbb{R}$.

Step 2. Now, since $\mathbf{u}_{*}^{\prime}( \pm \infty)=0$, there exists a large positive constant $M_{2}>0$ such that

$$
-2 \sigma \mathbf{u}_{*}^{\prime}(\xi) \leq 1, \text { if }|\xi| \geq M_{2}
$$

with $\sigma>0$ given in (3.12). Then by continuity of both $\mathbf{u}_{*}$ and $\hat{\mathbf{u}}$, and the fact that $\mathbf{u}_{*}(x+\bar{z})<\hat{\mathbf{u}}(x)$, there exists a small constant $\hat{h} \in\left(0, \frac{1}{2 \sigma}\right]$ such that

$$
\mathbf{u}_{*}(x+\bar{z}-2 \sigma \hat{h})<\hat{\mathbf{u}}(x), \quad x \in\left[-M_{2}-1-\bar{z}, M_{2}+1-\bar{z}\right] .
$$


As a consequence, when $|x+\bar{z}| \geq M_{2}+1$,

$$
\mathbf{u}_{*}(x+\bar{z}-2 \sigma \hat{h})-\hat{\mathbf{u}}(x)<\mathbf{u}_{*}(x+\bar{z}-2 \sigma \hat{h})-\mathbf{u}_{*}(x+\bar{z})=-2 \sigma \hat{h} \mathbf{u}_{*}^{\prime}(x+\bar{z}-2 \sigma \hat{h} \epsilon) \leq \hat{h},
$$

for some $\epsilon \in(0,1)$. As a consequence, we have

$$
\mathbf{u}_{*}(x+\bar{z}-2 \sigma \hat{h})-\hat{h} \leq \hat{\mathbf{u}}(x) \text { for all } x \in \mathbb{R},
$$

so that, using Lemma 3.1 and the comparison principle,

$$
\mathbf{u}_{*}\left(x-c_{*} t+\bar{z}-2 \sigma \hat{h}+\sigma \hat{h}\left(1-e^{-\beta t}\right)\right)-\hat{h} e^{-\beta t} \leq \hat{\mathbf{u}}\left(x-c_{*} t\right) \text { for all } x \in \mathbb{R} \text { and } t>0 .
$$

Then, keeping $\xi=x-c_{*} t$ fixed and sending $t \rightarrow+\infty$, we get

$$
\mathbf{u}_{*}(\xi+\bar{z}-\sigma \hat{h}) \leq \hat{\mathbf{u}}(\xi), \quad \forall \xi \in \mathbb{R} .
$$

This contradicts the minimality of $\bar{z}$ and thus $\mathbf{u}_{*}(x+\bar{z})=\hat{\mathbf{u}}(x)$ for all $x \in \mathbb{R}$, which concludes the proof of Theorem 2.

\section{Spectral analysis - Proof of Theorem 3}

Throughout this section, we will assume that both Hypotheses $(\mathrm{H} 1)-(\mathrm{H} 2 \eta)$ are satisfied and that there exists a unique (up to translation) traveling wave solution of (1.1) denoted $\mathfrak{u}^{*}(t)=\left(\mathbf{u}_{*}(n-\right.$ $\left.\left.c_{*} t\right)\right)_{n \in \mathbb{Z}}$ with $c_{*} \neq 0$. Without loss of generality, we shall assume that $c_{*}>0$. Let us recall that the profile $\mathbf{u}_{*} \in \mathscr{C}^{r+1}(\mathbb{R})$ and that is satisfies the limits

$$
\lim _{x \rightarrow-\infty} \mathbf{u}_{*}(x)=1 \text { and } \lim _{x \rightarrow+\infty} \mathbf{u}_{*}(x)=0,
$$

together with the property $\mathbf{u}_{*}^{\prime}<0$. We are interested by the spectral properties of the following linear operator $\mathcal{L}$ given by

$$
\mathcal{L} \mathbf{v}=c_{*} \mathbf{v}^{\prime}-\mathbf{v}+\mathcal{K}_{\delta} *\left[S^{\prime}\left(\mathbf{u}_{*}\right) \mathbf{v}\right] .
$$

We will consider $\mathcal{L}$ as an operator from $H^{1}(\mathbb{R})$ to $L^{2}(\mathbb{R})$. An easy preliminary result is the following.

Lemma 4.1. The operator $\mathcal{L}$ defined by (2.3) is the infinitesimal generator of a strongly continuous semigroup on $L^{2}(\mathbb{R})$.

Proof. This a consequence of the Hille-Yosida theorem and since $\mathcal{L}$ is closed with dense domain, it is enough to prove that its resolvent set contains of ray $\{\lambda \in(M,+\infty)\}$ with resolvent estimate

$$
\left\|(\lambda-\mathcal{L})^{-1}\right\| \leq \frac{1}{\lambda-M}, \text { for } \lambda>M .
$$

So assume that $(\lambda-\mathcal{L}) \mathbf{v}=\mathbf{w}$. Taking the inner product with $\mathbf{v}$, we find

$$
(\lambda+1)\|\mathbf{v}\|_{L^{2}(\mathbb{R})}^{2}-\int_{\mathbb{R}}\left(\sum_{j \in \mathbb{Z}} K_{j} S^{\prime}\left(\mathbf{u}_{*}(x-j)\right) \mathbf{v}(x-j)\right) \mathbf{v}(x) \mathrm{d} x=\langle\mathbf{v}, \mathbf{w}\rangle_{L^{2}(\mathbb{R})} .
$$


Using Cauchy-Schwartz inequality and the invariance of the $L^{2}$ norm under translation we get

$$
\left(\lambda+1-s_{m}\right)\|\mathbf{v}\|_{L^{2}(\mathbb{R})} \leq\|\mathbf{w}\|_{L^{2}(\mathbb{R})} .
$$

It is important to note that a direct consequence of the shift symmetry of the profile $\mathfrak{u} *(t)$

$$
\mathfrak{u}_{n}^{*}(t)=\mathfrak{u}_{n-1}^{*}\left(t-\frac{1}{c_{*}}\right), \quad n \in \mathbb{Z},
$$

is that the spectrum of $\mathcal{L}$ is invariant under the operation $\lambda \mapsto \lambda+2 \pi \mathbf{i} c_{*}$. This is reminiscent of the discrete invariance by translation of (1.1). Indeed, fix $p \in \mathbb{Z}$ and define $\omega=2 \pi \mathbf{i} p$ together with the exponential shift operator $e_{\omega}$

$$
e_{\omega} \mathbf{v}(x)=e^{\omega x} \mathbf{v}(x), \quad \forall x \in \mathbb{R} .
$$

Then, we have that

$$
\begin{aligned}
e_{-\omega} \mathcal{L} e_{\omega} \mathbf{v}(x) & =e^{-\omega x}\left(c_{*} \omega \mathbf{v}(x)+c_{*} \mathbf{v}^{\prime}(x)-\mathbf{v}(x)+\sum_{j \in \mathbb{Z}} K_{j} S^{\prime}\left(\mathbf{u}_{*}(x-j)\right) e^{\omega x} e^{-\omega j} \mathbf{v}(x-j)\right) \\
& =\left(\mathcal{L}+c_{*} \omega\right) \mathbf{v}(x),
\end{aligned}
$$

as $e^{-\omega j}=e^{-2 \pi \mathbf{i} p j}=1$ for all $(j, p) \in \mathbb{Z}^{2}$. Now, since $e_{ \pm \omega}$ are invertible operators on $H^{1}(\mathbb{R})$ and $L^{2}(\mathbb{R})$, we know that the spectrum $\sigma(\mathcal{L})$ of $\mathcal{L}$ equals that of $e_{-\omega} \mathcal{L} e_{\omega}$ and thus that of $\mathcal{L}+c_{*} \omega$. As $\mathcal{L} \mathbf{u}_{*}^{\prime}=0$, we automatically have that $2 \pi \mathbf{i} c_{*} \mathbb{Z} \subset \sigma(\mathcal{L})$.

With this shift symmetry in hand, we can return to the proof of Theorem 3. This will be accomplished by a series of Lemma essentially proving each assertions of the theorem.

Lemma 4.2. 0 is an eigenvalue of $\mathcal{L}$ with corresponding eigenvector $\mathbf{u}_{*}^{\prime}$ and it is geometrically and algebraically simple.

Proof. By translation invariance, we have that $\mathcal{L} \mathbf{u}_{*}^{\prime}=0$ with $\mathbf{u}_{*}^{\prime} \in H^{1}(\mathbb{R})$. Actually, we have that $\mathbf{u}_{*}^{\prime} \in \mathscr{C}_{b}^{r}(\mathbb{R})$ for $r \geq 2$ given in Hypothesis $(\mathrm{H} 1)$.

Let us first show that 0 is geometrically simple. Let suppose that $\mathcal{L} \mathbf{v}=0$ for some $\mathbf{v} \in H^{1}(\mathbb{R})$ such that $\mathbf{v}\left(x_{0}\right)<0$ for some $x_{0}$. For $\alpha>0$ we let $\mathbf{w}_{\alpha}=\mathbf{v}-\alpha \mathbf{u}_{*}^{\prime}$ and recall that $\mathbf{u}_{*}^{\prime}<0$. Let fix $M>0$ such that $x_{0} \in[-M, M]$. Then for all $\alpha$ large enough we have $\mathbf{w}_{\alpha}>0$ on $[-M, M]$ with $\mathcal{L} \mathbf{w}_{\alpha}=0$. As a consequence, we can use the comparison principle A.2 to the operator $\partial_{t}-\mathcal{L}$ and obtain that $\mathbf{w}_{\alpha}>0$ on $\mathbb{R}$. We denote $\bar{\alpha}=\inf \left\{\alpha \mid \mathbf{w}_{\alpha}>0\right.$ on $\left.\mathbb{R}\right\}$. We then have $\mathbf{w}_{\bar{\alpha}} \geq 0$ on $\mathbb{R}$ with some $\bar{x} \in \mathbb{R}$ where $\mathbf{w}_{\bar{\alpha}}^{\prime}(\bar{x})=\mathbf{w}_{\bar{\alpha}}(\bar{x})=0$. Thus,

$$
0=\mathcal{L} \mathbf{w}_{\bar{\alpha}}(\bar{x})=\sum_{j \in \mathbb{Z}} K_{j} S^{\prime}\left(\mathbf{u}_{*}(\bar{x}-j)\right) \mathbf{w}_{\bar{\alpha}}(\bar{x}-j),
$$


which implies that $\mathbf{w}_{\bar{\alpha}}(\bar{x}-j)=0$ where $K_{j}>0$ and by induction $\mathbf{w}_{\bar{\alpha}}(\bar{x}+n)=0$ for all $n \in \mathbb{Z}$. But now, the sequence $w_{n}(t)=\mathbf{w}_{\bar{\alpha}}\left(\bar{x}+n-c_{*} t\right)$ satisfies the initial value problem

$$
\begin{aligned}
& \dot{w}_{n}(t)=-w_{n}(t)+\sum_{j \in \mathbb{Z}} K_{j} S^{\prime}\left(\mathbf{u}_{*}\left(\bar{x}+n-j-c_{*} t\right)\right) w_{n-j}(t), \quad t>0 \text { and } n \in \mathbb{Z}, \\
& w_{n}(0)=0
\end{aligned}
$$

which admits the unique solution $w_{n}(t)=0$ and thus $\mathbf{w}_{\bar{\alpha}}\left(\bar{x}+n-c_{*} t\right)=0$ for all $t>0$ which implies that $\mathbf{w}_{\bar{\alpha}} \equiv 0$ and thus $v=\bar{\alpha} \mathbf{u}_{*}^{\prime}$.

Let us finally show that 0 is algebraically simple. Suppose that there exists $\mathbf{v} \in H^{1}(\mathbb{R})$ such that $\mathcal{L} \mathbf{v}=\mathbf{u}_{*}^{\prime}$ such that $\mathbf{v}\left(x_{0}\right)<0$ for some $x_{0}$. As above let us define $\mathbf{w}_{\alpha}=\mathbf{v}-\alpha \mathbf{u}_{*}^{\prime}$ and let $\alpha$ be large enough such that we have $\mathbf{w}_{\alpha}>0$ on $[-M, M]$ with $\left(\partial_{t}-\mathcal{L}\right) \mathbf{w}_{\alpha}=-u_{*}^{\prime}>0$ for all $x \in \mathbb{R}$. Thus, applying the comparison principle to $w_{\alpha}$ with the operator $\partial_{t}-\mathcal{L}$ we get that $\mathbf{w}_{\alpha}>0$ on $\mathbb{R}$. Let $\bar{\alpha}$ be the infimum of all such $\alpha$. Then we have $\mathbf{w}_{\bar{\alpha}} \geq 0$ on $\mathbb{R}$ with some $\bar{x} \in \mathbb{R}$ where $\mathbf{w}_{\bar{\alpha}}^{\prime}(\bar{x})=\mathbf{w}_{\bar{\alpha}}(\bar{x})=0$. As a consequence,

$$
0 \leq \sum_{j \in \mathbb{Z}} K_{j} S^{\prime}\left(\mathbf{u}_{*}(\bar{x}-j)\right) \mathbf{w}_{\bar{\alpha}}(\bar{x}-j)=\mathcal{L} \mathbf{w}_{\bar{\alpha}}(\bar{x})=\mathbf{u}_{*}^{\prime}(\bar{x})<0 .
$$

This gives a contradiction and thus $\mathbf{v} \equiv 0$ on $\mathbb{R}$.

Lemma 4.3. $\mathcal{L}: H^{1}(\mathbb{R}) \rightarrow L^{2}(\mathbb{R})$ is a Fredhlom operator of index 0 .

Proof. We are going to apply [16, Theorems $2 \& 3]$. To do so, we shall verify that all hypotheses of $[16$, Theorems $2 \& 3]$ are satisfied for the operator $\mathcal{T}: H^{1}(\mathbb{R}) \rightarrow L^{2}(\mathbb{R})$ defined as

$$
\mathcal{T} \mathbf{v}(x)=\frac{\mathrm{d}}{\mathrm{d} x} \mathbf{v}(x)-\sum_{j \in \mathbb{Z}} A_{j}(x) \mathbf{v}(x-j),
$$

where $A_{0}(x)=\left(1-K_{0} S^{\prime}\left(\mathbf{u}_{*}(x)\right)\right) / c_{*}$ and $A_{j}(x)=-K_{j} S^{\prime}\left(\mathbf{u}_{*}(x-j)\right) / c_{*}$ for all $j \neq 0$ and $x \in \mathbb{R}$. Let us note that $x \mapsto A_{j}(x) \in \mathscr{C}_{b}^{1}(\mathbb{R})$ for all $j \in \mathbb{Z}$ and that, because of Hypothesis $(\mathrm{H} 2 \eta)$, we have that

$$
\sum_{j \in \mathbb{Z}}\left\|A_{j}\right\|_{\mathscr{C}_{b}^{1}(\mathbb{R})} e^{\eta|j|} \leq C \sum_{j \in \mathbb{Z}} K_{j} e^{\eta|j|}<\infty
$$

for some constant $C>0$. Furthermore, there exists $A_{j}^{ \pm}=\lim _{x \rightarrow \pm \infty} A_{j}(x)$ where $A_{0}^{-}=\left(1-K_{0} S^{\prime}(1)\right) / c_{*}$, $A_{0}^{+}=\left(1-K_{0} S^{\prime}(0)\right) / c_{*}, A_{j}^{-}=-K_{j} S^{\prime}(1) / c_{*}$ and $A_{j}^{+}=-K_{j} S^{\prime}(1) / c_{*}$ for $j \neq 0$. And we also have that

$$
\sum_{j \in \mathbb{Z}}\left|A_{j}^{ \pm}\right| e^{\eta|j|}<\infty, \text { and } \lim _{x \rightarrow \pm \infty} \sum_{j \in \mathbb{Z}}\left|A_{j}(x)-A_{j}^{ \pm}\right| e^{\eta|j|}=0 .
$$

Note that in order to obtain the last equality, we have used the fact that

$$
A_{j}(x)-A_{j}^{-}=-\frac{K_{j}}{c_{*}} S^{\prime \prime}\left(\zeta_{j}(x)\right)\left(\mathbf{u}_{*}(x-j)-1\right) \text { for some } \zeta_{j}(x) \in\left(\mathbf{u}_{*}(x-j), 1\right)
$$


and remarked that $\left|A_{j}(x)-A_{j}^{-}\right| \leq s_{2} K_{j}$ for all $j \in \mathbb{Z}$ to be able to pass to the limit inside the infinite sum. It is also straightforward to check that

$$
\nu \mapsto \sum_{j} A_{j}^{ \pm} e^{\nu j},
$$

is a bounded analytic function in the stripe $\mathcal{S}_{\eta}=\{|\Re(\nu)|<\eta\}$ because of (H2 $\eta$ ). Finally, we need to show that for all $\ell \in \mathbb{R}$

$$
d^{ \pm}(\mathbf{i} \ell):=\mathbf{i} \ell-\sum_{j \in \mathbb{Z}} A_{j}^{ \pm} e^{-\mathbf{i} \ell j} \neq 0 .
$$

We only show that $d^{+}(\mathbf{i} \ell) \neq 0$, the other case being handled similarly. Suppose it is not the case, then there exists $\ell_{0} \in \mathbb{R}$ such that

$$
0=c_{*} \mathbf{i} \ell_{0}-1+S^{\prime}(0) \sum_{j \in \mathbb{Z}} K_{j} e^{-\mathbf{i} \ell_{0} j},
$$

and taking the real part we get that

$$
0=-1+S^{\prime}(0)+S^{\prime}(0) \sum_{j \in \mathbb{Z}} K_{j}\left(\cos \left(\ell_{0} j\right)-1\right) .
$$

But we have $0<S^{\prime}(0)<1$ and $K_{j}\left(\cos \left(\ell_{0} j\right)-1\right) \geq 0$ for all $j \in \mathbb{Z}$, a contradiction.

As a conclusion, we can apply [16, Theorem 2] which shows $\mathcal{T}: H^{1}(\mathbb{R}) \rightarrow L^{2}(\mathbb{R})$ is Fredholm and thus $\mathcal{L}: H^{1}(\mathbb{R}) \rightarrow L^{2}(\mathbb{R})$ is also Fredholm as $c_{*} \neq 0$. To compute the Fredholm index of $\mathcal{L}$, we rely on [16, Theorem 3] which states that the Fredholm index of $\mathcal{T}$ only depends on the limiting operators $\mathcal{T}^{ \pm}$defined by $\mathcal{T}^{ \pm} \mathbf{v}=\mathbf{v}^{\prime}-\sum_{j \in \mathbb{Z}} A_{j}^{ \pm} \mathbf{v}(\cdot-j)$. Moreover, the spectral flow formula (see [16, Theorem 2]) states that the Fredholm index of $T$ is gevin by the formula

$$
\operatorname{ind} \mathcal{T}=-\operatorname{cross}\left(\mathcal{T}^{\rho}\right)
$$

where $\mathcal{T}^{\rho}$, defined by $\mathcal{T}^{\rho} \mathbf{v}:=\mathbf{v}^{\prime}-\sum_{j \in \mathbb{Z}} A_{j}^{\rho} \mathbf{v}(\cdot-j)$, is a generic homotopy of constant operators joining $\mathcal{T}^{-}$at $\rho=-1$ and $\mathcal{T}^{+}$at $\rho=+1$ and $\operatorname{cross}\left(\mathcal{T}^{\rho}\right)$ counts the number of roots of the characteristic equation $d^{\rho}(z):=z-\sum_{j \in \mathbb{Z}} A_{j}^{\rho} e^{-z j}=0$ which cross the imaginary axis along this homotopy. Here, we consider $\mathcal{T}^{\rho}=\left((1-\rho) \mathcal{T}^{-}+(1+\rho) \mathcal{T}^{+}\right) / 2$ for $\rho \in[-1,1]$. It is clear that there exists no root of $d^{\rho}(z)=0$ on the imaginary axis for all $\rho \in[-1,1]$. Indeed, if there exists $\ell_{0} \in \mathbb{R}$ such that $d^{\rho}\left(\mathbf{i} \ell_{0}\right)=0$ then

$$
\begin{aligned}
0= & -2+\left((1-\rho) S^{\prime}(1)+(1+\rho) S^{\prime}(0)\right) \sum_{j \in \mathbb{Z}} K_{j} \cos \left(\ell_{0} j\right) \\
= & (1-\rho)\left(-1+S^{\prime}(1)+S^{\prime}(1) \sum_{j \in \mathbb{Z}} K_{j}\left(\cos \left(\ell_{0} j\right)-1\right)\right) \\
& +(1+\rho)\left(-1+S^{\prime}(0)+S^{\prime}(0) \sum_{j \in \mathbb{Z}} K_{j}\left(\cos \left(\ell_{0} j\right)-1\right)\right) \\
& <0,
\end{aligned}
$$

a contradiction. As a conclusion, we have ind $\mathcal{L}=\operatorname{ind} \mathcal{T}=0$. 
We define $\mathcal{L}^{*}: H^{1}(\mathbb{R}) \rightarrow L^{2}(\mathbb{R})$ as the formal adjoint of $\mathcal{L}$ by

$$
\mathcal{L}^{*} \mathbf{v}=-c_{*} \mathbf{v}-\mathbf{v}+S^{\prime}\left(\mathbf{u}_{*}\right) \mathcal{K}_{\delta} * \mathbf{v} .
$$

Corollary 4.4. The adjoint operator $\mathcal{L}^{*}: H^{1}(\mathbb{R}) \rightarrow L^{2}(\mathbb{R})$ has a negative eigenvector $\mathbf{q} \in H^{1}(\mathbb{R})$ corresponding to the simple eigenvalue 0 , i.e. $\operatorname{dim} \operatorname{ker} \mathcal{L}^{*}=1$.

Proof. Since 0 is a simple eigenvalue of $\mathcal{L}$ and $\mathcal{L}$ is Fredholm of index 0 , we readily have that $\operatorname{dim} \operatorname{ker} \mathcal{L}^{*}=1$ and thus $\mathcal{L}^{*} \mathbf{v}=0$ as a nonzero solution in $H^{1}(\mathbb{R})$. We only need to show that $\mathbf{v}$ has a sign. Suppose that $\mathbf{v}$ changes sign. Then one can find $f \in L^{2}(\mathbb{R})$ such that $\langle f, \mathbf{v}\rangle=0$ with $f<0$ on $\mathbb{R}$. Then, by the Fredholm alternative, the equation $\mathcal{L} \mathbf{u}=f$ has a solution $\mathbf{u}$. As before, we can choose $\alpha$ such that $\mathbf{w}_{\alpha}=\mathbf{u}-\alpha \mathbf{u}_{*}^{\prime} \geq 0$ on $\mathbb{R}$ and $\mathbf{w}_{\alpha}^{\prime}\left(x_{0}\right)=\mathbf{w}_{\alpha}\left(x_{0}\right)=0$ for some $x_{0}$. Then we have

$$
0 \leq \sum_{j \in \mathbb{Z}} K_{j} S^{\prime}\left(\mathbf{u}_{*}\left(x_{0}-j\right)\right) \mathbf{w}_{\alpha}\left(x_{0}-j\right)=\mathcal{L} \mathbf{w}_{\alpha}\left(x_{0}\right)=f\left(x_{0}\right)<0 .
$$

As a conclusion $\mathbf{v}$ has a sign which we choose to be negative.

Corollary 4.5. We have that $\sigma_{\text {ess }}(\mathcal{L}) \subset\{\lambda \mid \Re(\lambda) \leq-2 \beta\}$ where $\beta=\frac{1}{2} \min \left\{1-S^{\prime}(0), 1-S^{\prime}(1)\right\}>$ 0 .

Proof. Using similar argument, one can actually show that $\mathcal{L}-\lambda: H^{1}(\mathbb{R}) \rightarrow L^{2}(\mathbb{R})$ is Fredholm, with Fredholm index 0 , as long as $\Re(\lambda)>-2 \beta$, which gives the conclusion. Indeed, the essential spectrum of $\mathcal{L}$ is the set of all $\lambda \in \mathbb{C}$ such that either $\mathcal{L}-\lambda$ is not Fredholm or $\mathcal{L}-\lambda$ is Fredholm, but $\operatorname{ind}(\mathcal{L}-\lambda) \neq 0$.

Lemma 4.6. There exist $\eta_{*}, \eta_{* *} \in(0, \eta)$ and some constants $C_{*}>0, C_{* *}>0$ such that

$$
\left|\mathbf{u}_{*}^{\prime}(x)\right| \leq C_{*} e^{-\eta_{*}|x|}\left\|\mathbf{u}_{*}^{\prime}\right\|_{L^{\infty}(\mathbb{R})}, \text { and }|\mathbf{q}(x)| \leq C_{* *} e^{-\eta_{* *}|x|}\|\mathbf{q}\|_{L^{\infty}(\mathbb{R})},
$$

for all $x \in \mathbb{R}$.

Proof. We have proved in the previous lemma that $d^{ \pm}(\mathbf{i} \ell) \neq 0$ for all $\ell \in \mathbb{R}$ and $\mathcal{L}$ is then asymptotically hyperbolic in the sens of Malet-Parret [9, 22]. Hence, we obtain from [9, Lemma 4.3] that there exist constants $\eta_{*} \in(0, \eta)$ and $C_{*}>0$ for which

$$
|\mathbf{v}(x)| \leq C_{*} e^{-\eta_{*}|x|}\|\mathbf{u}\|_{L^{\infty}(\mathbb{R})}+C_{*} \int_{\mathbb{R}} e^{-\eta_{*}|x-y|}|\mathbf{w}(y)| \mathrm{d} y, \quad x \in \mathbb{R},
$$

holds for each $\mathbf{v} \in H^{1}(\mathbb{R})$, where $\mathbf{w}=\mathcal{L} \mathbf{v}$. Since $\mathcal{L} \mathbf{u}_{*}^{\prime}=0$ we conclude that

$$
\left|\mathbf{u}_{*}^{\prime}(x)\right| \leq C_{*} e^{-\eta_{*}|x|}\left\|\mathbf{u}_{*}^{\prime}\right\|_{L^{\infty}(\mathbb{R})}, \quad x \in \mathbb{R} .
$$

As $\mathcal{L}^{*}$ is also asymptotically hyperbolic and since $\mathcal{L}^{*} \mathbf{q}=0$, we get

$$
|\mathbf{q}(x)| \leq C_{* *} e^{-\eta_{* *}|x|}\|\mathbf{q}\|_{L^{\infty}(\mathbb{R})},
$$

for all $x \in \mathbb{R}$. 
Lemma 4.7. We have $\left\{\lambda \mid \Re(\lambda) \geq 0, \lambda \neq 2 \pi \mathbf{i} c_{*} p, p \in \mathbb{Z}\right\} \subset \rho(\mathcal{L})$ where $\rho(\mathcal{L})$ is the resolvent set of $\mathcal{L}$.

Proof. Suppose that $\lambda=\lambda_{1}+\mathbf{i} \lambda_{2}$ with $\lambda_{1} \geq 0$ and $\lambda_{2} \neq 2 \pi \mathbf{i} c_{*} p$ for $p \in \mathbb{Z}$ is an eigenvalue with a corresponding eigenfunction $\mathbf{u}=\mathbf{u}_{1}+\mathbf{i} \mathbf{u}_{2} \neq 0$. Without loss of generality, we may assume that $0<\lambda_{2}<2 \pi \mathbf{i} c_{*}$, as the spectrum $\mathcal{L}$ is invariant under the translation $\lambda \mapsto \lambda+2 \pi \mathbf{i} c_{*}$. We consider the Cauchy problem

$$
\begin{aligned}
\partial_{t} \mathbf{v} & =\mathcal{L} \mathbf{v}-\lambda_{1} \mathbf{v}, \\
v(0) & =\mathbf{u}_{1}
\end{aligned}
$$

which has a solution $\mathbf{v}(x, t)=\mathbf{u}_{1}(x) \cos \left(\lambda_{2} t\right)-\mathbf{u}_{2}(x) \sin \left(\lambda_{2} t\right)$. Note that $\mathbf{v}(x, t) \leq|\mathbf{u}(x)|$ for all $x \in \mathbb{R}$ and $t \geq 0$. We claim that there $\alpha>0$ such that $\mathbf{v}(x, t) \leq-\alpha \mathbf{u}_{*}^{\prime}(x)$ holds for all $x \in \mathbb{R}$ and $t \geq 0$. To prove the claim, we follow the strategy in [3] and let $\theta_{0}$ be a constant such that $0<\theta_{0}<\min \left\{1-S^{\prime}(0), 1-S^{\prime}(1)\right\}$. Then we can choose $M>0$ large enough such that

$$
\theta_{0}-1+\sum_{j \in \mathbb{Z}} K_{j} S^{\prime}\left(\mathbf{u}_{*}(x-j)\right) \leq 0, \text { for all }|x| \geq M
$$

and such that $\left|\mathbf{u}\left(x_{0}\right)\right|>0$ for some $x_{0} \in[-M, M]$. Since, $\mathbf{u}_{*}<0$ we can always find $\alpha>0$ such that $|\mathbf{u}(x)| \leq-\alpha \mathbf{u}_{*}^{\prime}(x)$ for $|x| \leq M$. We prove that the claim holds with this choice of $\alpha$. Since $|\mathbf{u}(x)| \rightarrow 0$ as $x \rightarrow \pm \infty$, there exists $\epsilon>0$ such that $\mathbf{v}(x, t) \leq-\alpha \mathbf{u}_{*}^{\prime}(x)+\epsilon$ for all $x \in \mathbb{R}$ and $t \geq 0$ and let $\epsilon_{0} \geq 0$ be the infimum of such $\epsilon$. We prove that $\epsilon_{0}=0$. Consider the function $\mathbf{w}(x, t)=-\alpha \mathbf{u}_{*}^{\prime}(x)+\epsilon_{0} e^{-\theta_{0} t}$. We have

$\partial_{t} \mathbf{w}-\mathcal{L} \mathbf{w}+\lambda_{1} \mathbf{w}=-\epsilon_{0} e^{-\theta_{0} t}\left(\theta_{0}-1+\sum_{j \in \mathbb{Z}} K_{j} S^{\prime}\left(\mathbf{u}_{*}(x-j)\right)\right)+\lambda_{1} \mathbf{w} \geq 0$, for all $|x| \geq M$ and $t>0$.

Therefore, $\mathbf{v}$ is a super solution of (4.2) on $|x|>M$ and notice that $\mathbf{v}(x, t) \leq|\mathbf{u}(x)| \leq-\alpha \mathbf{u}_{*}^{\prime}(x) \leq$ $\mathbf{w}(x, t)$ on $|x| \leq M$ and that $\mathbf{v}(x, 0)=\mathbf{u}_{1}(x) \leq|\mathbf{u}(x)| \leq-\alpha \mathbf{u}_{*}^{\prime}(x)+\epsilon_{0}=\mathbf{w}(x, 0)$ for all $x \in \mathbb{R}$. Then, the comparison principle gives $\mathbf{v}(x, t) \leq \mathbf{w}(x, t)$ for all $x \in \mathbb{R}$ and all $t>0$. Therefore, for all positive integer $p>0$, we have

$$
\mathbf{v}(x, t)=\mathbf{v}\left(x, t+2 p \pi / \lambda_{2}\right) \leq-\alpha \mathbf{u}_{*}^{\prime}(x)+\epsilon_{0} e^{-\theta_{0}\left(t+2 p \pi / \lambda_{2}\right)} \underset{p \rightarrow+\infty}{\longrightarrow \rightarrow}-\alpha \mathbf{u}_{*}^{\prime}(x),
$$

and thus $\epsilon_{0}=0$ and the claim is proved.

Now, we pick $\bar{\alpha}>0$ such that $|\mathbf{u}(x)| \leq-\bar{\alpha} \mathbf{u}_{*}^{\prime}(x)$ for all $|x| \leq M$ with some $\bar{x}$ such that $|\mathbf{u}(\bar{x})|=$ $-\bar{\alpha} \mathbf{u}_{*}^{\prime}(\bar{x})>0$ and using the comparison principle we deduce that $\mathbf{v}(x, t)<-\bar{\alpha} \mathbf{u}_{*}^{\prime}(x)$ for all $x \in \mathbb{R}$ and $t>0$. If we choose $\bar{t}$ such that $\mathbf{u}(\bar{x}) /|\mathbf{u}(\bar{x})|=e^{-\mathbf{i} \lambda_{2} \bar{t}}$, then $\mathbf{v}(\bar{x}, \bar{t})=|\mathbf{u}(\bar{x})|=-\bar{\alpha} \mathbf{u}_{*}^{\prime}(\bar{x})>\mathbf{v}(\bar{x}, \bar{t})$, which is a contradiction. Therefore $\mathbf{u} \equiv 0$ and $\lambda$ is not an eigenvalue.

Finally, assume that $\lambda>0$ is an eigenvalue with a real eigenfunction $\mathbf{u}$ that possesses a point where it is positive. Then $\mathbf{v}(x, t)=\mathbf{u}(x)$ is a solution of (4.2) with $\lambda_{1}=\lambda>0$ and initial condition $\mathbf{v}(x, 0)=\mathbf{u}(x)$. A similar argument as we did before can be used to prove that $\mathbf{u} \equiv 0$ and so $\lambda>0$ is not an eigenvalue. 
We are now ready to conclude the proof of Theorem 3.

Proof of Theorem 3. Only assertion (iii) of the theorem remains to be proved. From the previous lemma, we have $\left\{\lambda \mid \Re(\lambda) \geq 0, \lambda \neq 2 \pi \mathbf{i} c_{*} p, p \in \mathbb{Z}\right\} \subset \rho(\mathcal{L})$ and we would like to extend the resolvent set to the stripe $S_{\kappa}:=\{\lambda \mid-\kappa \leq \Re(\lambda)<0\}$ for $\kappa<2 \beta$. As $\mathcal{L}-\lambda: H^{1}(\mathbb{R}) \rightarrow L^{2}(\mathbb{R})$ is Fredholm index 0 for $\Re(\lambda)>-2 \beta$ it will be enough to show that $\mathcal{L}-\lambda$ is injective in the stripe $S_{\kappa}$. We claim that there exists $\lambda_{i}>0$ such that for all $|\Im(\lambda)|>\lambda_{i}$ with $-\kappa \leq \Re(\lambda)<0, \mathcal{L}-\lambda$ is injective. If the claim holds true, then suppose by contradiction that $\mathcal{L}$ has an eigenvalue $\lambda_{0}$ within the rectangle $\{\lambda \mid-\kappa \leq \Re(\lambda)<0\} \cap\left\{\lambda \mid \Im(\lambda) \leq c_{*} \pi\right\}$. Then, by the shift symmetry, there exists $p_{0}>0$ large enough such that $\Im\left(\lambda_{0}\right)+2 \pi c_{*} p_{0}>\lambda_{i}$. As consequence, $\mathcal{L}-\lambda_{0}-2 \pi c_{*} p_{0} \mathbf{i}$ is not injective, as it has a kernel, and we reached a contradiction. To conclude the proof, it only remains to prove the claim. We thus suppose that there exists $\mathbf{u} \in H^{1}(\mathbb{R})$ such that $(\mathcal{L}-\lambda) \mathbf{u}=0$. First, we rescale this eigenvalue problem by introducing a new variable $z=\Im(\lambda) x$ and a new unknown $\tilde{\mathbf{u}}$, such that we obtain

$$
c_{*} \frac{\mathrm{d} \tilde{\mathbf{u}}(z)}{\mathrm{d} z}+\mathbf{i} \tilde{\mathbf{u}}(z)+\frac{s_{m}}{\Im(\lambda)}\left(-\tilde{\mathbf{u}}(z)+\sum_{j \in \mathbb{Z}} K_{j} \tilde{\mathbf{u}}(z-\Im(\lambda) j)\right)=\frac{1}{\Im(\lambda)} \mathcal{R}(z, \Re(\lambda), \tilde{\mathbf{u}}),
$$

whenever $\Im(\lambda) \neq 0$ and $\mathcal{R}(z, \Re(\lambda), \tilde{\mathbf{u}})$ collects all the remainder terms and satisfies the estimates:

$$
\|\mathcal{R}(z, \Re(\lambda), \tilde{\mathbf{u}})\|_{L^{2}(\mathbb{R})} \leq(|\Re(\lambda)|+C)\|\tilde{\mathbf{u}}\|_{L^{2}(\mathbb{R})},
$$

for some constant $C>0$ independent of $\lambda$. Let us define the operator $\mathcal{H}_{\lambda}: H^{1}(\mathbb{R}) \rightarrow L^{2}(\mathbb{R})$ by

$$
\mathcal{H}_{\lambda} \mathbf{v}(x):=c_{*} \mathbf{v}^{\prime}(x)+\mathbf{i v}(x)+\frac{s_{m}}{\Im(\lambda)}\left(-\mathbf{v}(x)+\sum_{j \in \mathbb{Z}} K_{j} \mathbf{v}(x-\Im(\lambda) j)\right),
$$

which has Fourier symbol

$$
\widehat{\mathcal{H}_{\lambda}}(\mathbf{i} \ell)=c_{*} \mathbf{i} \ell+\mathbf{i}+\frac{s_{m}}{\Im(\lambda)}\left(\sum_{j \in \mathbb{Z}} K_{j}(\cos (\ell \Im(\lambda) j)-1)\right), \quad \ell \in \mathbb{R} .
$$

Let $\lambda_{*}>0$ be fixed. We would like to show that there exists a constant $\tilde{C}>0$, such that $\left|\widehat{\mathcal{H}_{\lambda}}(\mathbf{i} \ell)\right| \geq \tilde{C}$ for all $\ell \in \mathbb{R}$ and all $\lambda \in \mathbb{C}$ with $|\Im(\lambda)|>\lambda_{*}$. Let us first remark that

$$
\left|\widehat{\mathcal{H}_{\lambda}}(\mathbf{i} \ell)\right|=\left|c_{*} \ell+1\right|+\frac{s_{m}}{|\Im(\lambda)|} A(\ell \Im(\lambda)),
$$

where we set $A(y):=\sum_{j \in \mathbb{Z}} K_{j}(1-\cos (y j))$. We see that $A(y)>0$ for any $y \in(0,2 \pi)$. Because of the symmetry $K_{j}=K_{-j}$, we have $A^{\prime}(0)=0$ and $A^{\prime \prime}(0)=\sum_{j} K_{j} j^{2}>0$. As a consequence, there exists some constant $d_{0}>0$ such that $A(y)>d_{0} y^{2}$ for all $y \in(0,2 \pi)$. To conclude that $\left|\widehat{\mathcal{H}_{\lambda}}(\mathbf{i} \ell)\right|$ is bounded away from 0 we argue as follows. Suppose that $\left|\Im\left(\widehat{\mathcal{H}_{\lambda}}(\mathbf{i} \ell)\right)\right|=\left|c_{*} \ell+1\right|<1 / 2$, then we have $1 / 2=1-1 / 2 \leq\left|c_{*} \ell\right|$ and thus $|\ell| \geq 1 / 2 c_{*}$. Now in that case, we observe that

$$
\left|\Re\left(\widehat{\mathcal{H}_{\lambda}}(\mathbf{i} \ell)\right)\right|=\frac{s_{m}}{|\Im(\lambda)|} A(\ell \Im(\lambda))=\frac{s_{m}(\ell \Im(\lambda))^{2}}{|\Im(\lambda)|(\ell \Im(\lambda))^{2}} A(\ell \Im(\lambda)) \geq \frac{d_{0} s_{m} \lambda_{*}}{4 c_{*}^{2}} .
$$


Coming back to (4.3), we have

$$
\mathcal{H}_{\lambda} \tilde{\mathbf{u}}=\frac{1}{\Im(\lambda)} \mathcal{R}(z, \Re(\lambda), \tilde{\mathbf{u}}),
$$

and as a consequence, using the bound in (4.4) and the fact that $\left|\widehat{\mathcal{H}_{\lambda}}(\mathbf{i} \ell)\right|$ is bounded away from 0 for all $\ell \in \mathbb{R}$ and all $\lambda \in \mathbb{C}$ with $|\Im(\lambda)|>\lambda_{*}$, we have

$$
\|\tilde{\mathbf{u}}\|_{L^{2}(\mathbb{R})} \leq \frac{(|\Re(\lambda)|+C)}{\tilde{C}|\Im(\lambda)|}\|\tilde{\mathbf{u}}\|_{L^{2}(\mathbb{R})} .
$$

Recall that $|\Re(\lambda)| \leq \kappa$, then for all $\lambda \in \mathbb{C}$ such that $|\Im(\lambda)|>\lambda_{i}:=\max \left\{2\left(\frac{\kappa+C}{\tilde{C}}\right), \lambda_{*}\right\}$, the only solution of (4.3) has to be $\tilde{\mathbf{u}}=0$. As a consequence, we have shown that $\mathcal{L}-\lambda: H^{1}(\mathbb{R}) \rightarrow L^{2}(\mathbb{R})$ is injective for all $|\Im(\lambda)|>\lambda_{i}$ with $-\kappa \leq \Re(\lambda)<0$. This concludes the proof of the claim and thus of the theorem.

\section{Discussion}

Summary of main results. In this paper, we have shown the existence of monotone traveling front solutions for lattice neural field equations of the form

$$
\dot{u}_{n}(t)=-u_{n}(t)+\sum_{j \in \mathbb{Z}} K_{j} S\left(u_{n-j}(t)\right)
$$

with infinite range couplings and when the associated dynamics of each individual neuron is of bistable type, under mild assumptions on $\left(K_{j}\right)_{j \in \mathbb{Z}}$ and $S$. We also proved that traveling front solutions having nonzero wave speed are unique up to translation. Furthermore, we have explored the spectral properties of such nonzero wave speed fronts, provided an extra exponential localization of the connectivity weights $K_{j}$. More precisely, we have interpreted solutions of the LNFEs as solutions to the following nonlocal partial differential equation

$$
\partial_{t} \mathbf{u}(t, x)=c_{*} \partial_{x} \mathbf{u}(t, x)-\mathbf{u}(t, x)+\int_{\mathbb{R}} K_{j} S(\mathbf{u}(t, x-j)),
$$

and we have studied spectral properties of the linearized operator $\mathcal{L}$ given by

$$
\mathcal{L} \mathbf{v}=c_{*} \partial_{x} \mathbf{v}-\mathbf{v}+\mathcal{K}_{\delta} *\left[S^{\prime}\left(\mathbf{u}_{*}\right) \mathbf{v}\right]
$$

round a non zero wave speed traveling front $\left(\mathbf{u}_{*}, c_{*}\right)$. Because of the invariance by translation, $\lambda=0$ is an algebraically simple eigenvalue of $\mathcal{L}$ associated to the eigenvector $\mathbf{u}_{*}^{\prime}$ and the spectrum of $\mathcal{L}$ is invariant under the operation $\lambda \mapsto \lambda+2 \pi \mathbf{i} c_{*}$ such that $2 \pi \mathbf{i} c_{*} \mathbb{Z} \subset \sigma(\mathcal{L})$. The main result regarding the operator $\mathcal{L}$ is that $\mathcal{L}-\lambda: H^{1}(\mathbb{R}) \rightarrow L^{2}(\mathbb{R})$ is invertible for all $\lambda \in \mathbb{C} \backslash 2 \pi \mathbf{i} c_{*} \mathbb{Z}$ such that $\Re(\lambda) \geq-\kappa>\min \left\{-1+S^{\prime}(0),-1+S^{\prime}(1)\right\}$. 
Towards nonlinear stability. Our spectral analysis is a preliminary step towards a nonlinear stability analysis of (1.1) around a traveling front solution. Let us explain how such a spectral analysis could be used to get insight on the asymptotic behavior of solutions of (1.1) starting from an initial condition close to a traveling front solution. Let us introduce the nonlinear operator

$$
\begin{aligned}
\mathcal{F}: \ell^{\infty}(\mathbb{R}) & \longrightarrow \ell^{\infty}(\mathbb{R}) \\
\mathfrak{u} & \longmapsto \mathcal{F}(\mathfrak{u})=-\mathfrak{u}+\mathcal{K} *_{d} S(\mathfrak{u}),
\end{aligned}
$$

where for all $n \in \mathbb{Z}$ we have set

$$
\left(\mathcal{K} *_{d} S(\mathfrak{u})\right)_{n}:=\sum_{j \in \mathbb{Z}} K_{j} S\left(\mathfrak{u}_{n-j}\right)
$$

and

$$
\ell^{\infty}(\mathbb{R}):=\left\{\mathfrak{u}=\left(\mathfrak{u}_{n}\right)_{n \in \mathbb{Z}} \in \mathbb{R}^{\mathbb{Z}}\left|\|\mathfrak{u}\|_{\ell^{\infty}(\mathbb{R})}:=\sup _{n \in \mathbb{Z}}\right| \mathfrak{u}_{n} \mid<\infty\right\} .
$$

Using this notation, we can then write (1.1) as

$$
\dot{\mathfrak{u}}(t)=\mathcal{F}(\mathfrak{u}(t)), \quad t>0 .
$$

for which $\mathfrak{u}^{*}(t)=\left(\mathbf{u}_{*}\left(n-c_{*} t\right)\right)_{n \in \mathbb{Z}}$ with $c_{*} \neq 0$ is a solution, where the existence of the profile $\mathbf{u}_{*}$ is given by Theorems 1 and 2. To study the stability of $\mathfrak{u}^{*}(t)$, we look for solutions of (5.2) that can be written as $\mathfrak{u}(t)=\mathfrak{u}^{*}(t)+\mathfrak{v}(t)$ where $\mathfrak{v}(t)$ is a perturbation of the traveling wave solution $\mathfrak{u}^{*}(t)$. We then find that $\mathfrak{v}(t)$ must satisfy the time-dependent lattice neural field equation

$$
\dot{\mathfrak{v}}(t)=D \mathcal{F}\left(\mathfrak{u}^{*}(t)\right) \mathfrak{v}(t)+\mathcal{N}(t, \mathfrak{v}(t)),
$$

in which

$$
\mathcal{N}(t, \mathfrak{v}(t))=\mathcal{F}\left(\mathfrak{u}^{*}(t)+\mathfrak{v}(t)\right)-\mathcal{F}\left(\mathfrak{u}^{*}(t)\right)-D \mathcal{F}\left(\mathfrak{u}^{*}(t)\right) \mathfrak{v}(t) .
$$

Then the strategy would be to obtain spectral properties for $\dot{\mathfrak{v}}(t)=D \mathcal{F}\left(\mathfrak{u}^{*}(t)\right) \mathfrak{v}(t)$ from those of the operator $\mathcal{L}$ in order to be able to close a nonlinear stability argument. This method was introduced and successfully implemented by Benzoni-Gavage and coauthors in [6] by analyzing associated Green's functions for the nonlinear stability analysis of semidiscrete shock waves and more recently reused in the context of nonlinear stability analysis of traveling pulses in the discrete FitzHugh-Nagumo equations with finite and infinite range interactions [20, 25]. We conjecture the following result with perturbations measured in the Banach spaces $\ell^{p}(\mathbb{R})$, which are defined by

$$
\ell^{p}(\mathbb{R}):=\left\{\mathfrak{u}=\left(\mathfrak{u}_{n}\right)_{n \in \mathbb{Z}} \in \mathbb{R}^{\mathbb{Z}} \mid\|\mathfrak{u}\|_{\ell^{p}(\mathbb{R})}:=\left(\sum_{n \in \mathbb{Z}}\left|\mathfrak{u}_{n}\right|^{p}\right)^{\frac{1}{p}}<\infty\right\}
$$

for $1 \leq p<\infty$.

Conjecture 1 (Nonlinear stability). Suppose that the Hypotheses (H1)-(H2ף) are satisfied and let $\left(\mathbf{u}_{*}, c_{*}\right)$, with $c_{*} \neq 0$, be the unique (up to translation) strictly monotone traveling wave solution to (1.1) as given in Theorem 1. We denote by $\mathfrak{u}^{*}(t):=\left(\mathbf{u}_{*}\left(n-c_{*} t\right)\right)_{n \in \mathbb{Z}}$. Then for all $1 \leq p \leq \infty$, 
there exist constants $\delta>0, C>0, \omega>0$ such that for all sequences $\mathfrak{u}^{0}=\left(u_{n}^{0}\right)_{n \in \mathbb{Z}}$ which satisfy $\left\|\mathfrak{u}^{0}-\mathfrak{u}^{*}(0)\right\|_{\ell^{p}(\mathbb{R})} \leq \delta$, there exists an asymptotic phase shift $\xi_{0} \in \mathbb{R}$ such that the unique solution $t \rightarrow \mathfrak{u}(t)=\left(u_{n}(t)\right)_{n \in \mathbb{Z}}$ of $(1.1)$, with initial condition $\mathfrak{u}(0)=\mathfrak{u}^{0}$, verifies

$$
\left\|\mathfrak{u}(t)-\mathfrak{u}^{*}\left(t+\xi_{0}\right)\right\|_{\ell^{p}(\mathbb{R})} \leq C e^{-\omega t}\left\|\mathfrak{u}^{0}-\overline{\mathfrak{u}}(0)\right\|_{\ell^{p}(\mathbb{R})},
$$

for all $t \geq 0$.

Monostable nonlinearities. An interesting extension of the present work would be to look at another class of nonlinearity. Here, we assumed that the associated dynamics of each individual neuron is of bistable type, but one could study monostable type of dynamics. Roughly speaking, in the monostable case, one would suppose that for $f$ defined in $(2.2)$ we have $f(0)=f(1)=0$ and $f(u)>0$ for all $u \in(0,1)$ with $f^{\prime}(0)>0$ and $f^{\prime}(1)<0$. Such a case was recently considered in [14] for continuous neural field equations and we expect to find similar results in the discrete setting. In particular, when the connectivity weights $K_{j}$ are not exponentially localized in the sens of Hypothesis $(\mathrm{H} 2 \eta)$, we conjecture that there does not exist any monotone traveling front solutions and that the level sets of solutions of the LNFE associated to a compactly supported initial condition propagate with an infinite asymptotic speed. This will be the subject of futur works.

Linear adaptation \& traveling pulses. To study the propagation of pulses, i.e. non-monotone traveling waves connecting the homogeneous stationary states, one introduces a linear adaptation mechanism so that the LNFE becomes a system of the form

$$
\begin{aligned}
& \dot{u}_{n}(t)=-u_{n}(t)+\sum_{j \in \mathbb{Z}} K_{j} S\left(u_{n-j}(t)\right)-v_{n}(t), \\
& \dot{v}_{n}(t)=\epsilon\left(u_{n}(t)-a v_{n}(t)\right),
\end{aligned}
$$

where $0<\epsilon \ll 1$ is a small temporal parameter reflecting the slow nature of the adaptation mechanism and $a>0$ is a fixed parameter chosen such that $(u, v)=(0,0)$ is the only stationary homogeneous steady state of (5.5). Proving the existence of nonzero wave speed traveling pulse solutions of $(5.5)$ amounts at finding profiles $\mathbf{u} \in \mathscr{C}^{1}(\mathbb{R})$ and $\mathbf{v} \in \mathscr{C}^{1}(\mathbb{R})$ together with a real $c \neq 0$ solution of

$$
\begin{aligned}
& -c \mathbf{u}^{\prime}=-\mathbf{u}+\mathcal{K} *_{\delta} S(\mathbf{u})-\mathbf{v}, \\
& -c \mathbf{v}^{\prime}=\epsilon(\mathbf{u}-a \mathbf{v}),
\end{aligned}
$$

verifying the limits

$$
\lim _{x \rightarrow \pm \infty}(\mathbf{u}(x), \mathbf{v}(x))=(0,0) .
$$

Suppose that Hypotheses (H1) and ( $\mathrm{H} 2 \eta)$ are satisfied and that there exists a unique (up to translation) traveling wave front solution $\mathbf{u}_{*}^{f}$ with nonzero wave speed $c_{*}>0$ of (5.6a) for $\mathbf{v}=0$ connecting 
$\mathbf{u}=0$ and $\mathbf{u}=1$ at $\mp \infty$, then the spectral properties of the linear operator $\mathcal{L}\left(\mathbf{u}_{*}^{f}\right)$ associated to this front (as defined in (2.3)) are given by Theorem 3. If we further suppose that there exists some $v_{*} \in \mathbb{R}$ for which there is a traveling back solution $\mathbf{u}_{*}^{b}$ of $(5.6 \mathrm{a})$ for $\mathbf{v}=v_{*}$ with precisely nonzero wave speed $c_{*}$, connecting two states $v_{ \pm}$at $\pm \infty$, we shall expect that the same spectral properties hold true for the linear operator $\mathcal{L}\left(\mathbf{u}_{*}^{b}\right)$ associated to this back solution. Under this setting, we can then rely on [17, Theorem 1] which tells us that for each sufficiently small $\epsilon>0$, there exists profiles $\mathbf{u}_{\epsilon}, \mathbf{v}_{\epsilon} \in \mathscr{C}^{1}(\mathbb{R})$ and a wave speed $c_{\epsilon}>0$ such that $\left(\mathbf{u}_{\epsilon}\left(n-c_{\epsilon} t\right), \mathbf{v}_{\epsilon}\left(n-c_{\epsilon} t\right)\right)$ is solution of (5.6) for all $n \in \mathbb{Z}$ and $t>0$ and further satisfy the limits

$$
\lim _{x \rightarrow \pm \infty}\left(\mathbf{u}_{\epsilon}(x), \mathbf{v}_{\epsilon}(x)\right)=(0,0) .
$$

As a consequence, upon assuming that $S$ (and thus $f$ in (2.2)) has the good properties to support the existence of a traveling back solution $\mathbf{u}_{*}^{b}$ whose associated spectral properties are given by Theorem 3 , then one gets the existence of traveling pulse solutions for the LNFE with linear adaptation $(5.5)$.

Approximation of continuous neural field equations. As explained in the introduction, LNFEs can be obtained by discretizing continuous neural field equations of the form

$$
\partial_{t} u(t, x)=-u(t, x)+\int_{\mathbb{R}} K(x-y) S(u(t, y)) \mathrm{d} y, \quad x \in \mathbb{R} .
$$

Assume a regular discretization of the real line with $x_{j}=j h$ for $j \in \mathbb{Z}$ and $0<h \ll 1$. Evaluating the above equation at $x=x_{n}$ and slightly rewriting the integral, we get

$$
\partial_{t} u\left(t, x_{n}\right)=-u\left(t, x_{n}\right)+\sum_{j \in \mathbb{Z}} \int_{j h}^{j h+h} K(y) S\left(u\left(t, x_{n}-y\right)\right) \mathrm{d} y,
$$

from which we can use for example a rectangle quadrature rule to approximate

$$
\int_{j h}^{j h+h} K(y) S\left(u\left(t, x_{n}-y\right)\right) \mathrm{d} y \approx h K\left(x_{j}\right) S\left(u\left(t, x_{n-j}\right)\right),
$$

Eventually, we arrive at the following LNFE for $u_{n}^{h}(t)$, an approximation of $u(t, n h)$,

$$
\dot{u}_{n}^{h}(t)=-u_{n}^{h}(t)+\sum_{j \in \mathbb{Z}} K_{j}^{h} S\left(u_{n-j}^{h}(t)\right),
$$

where we set $K_{j}^{h}:=h K(j h)$. One can then use the techniques in $[5,25]$ together with the results developed in this paper to study traveling front solutions of (5.7) and analyze under which assumptions the corresponding front solution converges as $h \rightarrow 0$ to an actual traveling wave solution of the initial continuous neural field equation.

Other types of networks. In a more exploratory direction, it would also be interesting to study neuronal dynamics set on other types of networks, such as for example homogeneous trees or ErdösRéyni graphs. Let us mention the works of $[18,21]$ and references therein on the study of traveling fronts for reaction-diffusion on networks. 


\section{Acknowledgements}

GF received support from the ANR project NONLOCAL ANR-14-CE25-0013.

\section{A Comparison principles}

In this section, we present comparison principles for the lattice neural field equation (1.1). For any sequence $\mathfrak{u}=\left(u_{n}\right)_{n \in \mathbb{Z}} \in \ell^{\infty}(\mathbb{R})$, we recall the definition of the nonlinear operator $\mathcal{F}$ :

$$
\begin{aligned}
\mathcal{F}: \ell^{\infty}(\mathbb{R}) & \longrightarrow \ell^{\infty}(\mathbb{R}) \\
\mathfrak{u} & \longmapsto \mathcal{F}(\mathfrak{u})=-\mathfrak{u}+\mathcal{K} *_{d} S(\mathfrak{u}),
\end{aligned}
$$

where for all $n \in \mathbb{Z}$ we have set

$$
\left(\mathcal{K} *_{d} S(\mathfrak{u})\right)_{n}:=\sum_{j \in \mathbb{Z}} K_{j} S\left(u_{n-j}\right) .
$$

Lemma A.1. Assume that $\mathcal{K}$ and $S$ satisfy Hypotheses (H1)-(H2). Suppose that there exist two sequences $t \mapsto \mathfrak{u}(t) \in \mathscr{C}^{1}\left(\mathbb{R}, \ell^{\infty}(\mathbb{R})\right)$ and $t \mapsto \mathfrak{v}(t) \in \mathscr{C}^{1}\left(\mathbb{R}, \ell^{\infty}(\mathbb{R})\right)$ that satisfy

$$
\dot{\mathfrak{u}}(t) \leq \mathcal{F}(\mathfrak{u}(t)), \quad \dot{\mathfrak{v}}(t) \geq \mathcal{F}(\mathfrak{v}(t)) \quad \forall t>0, \quad \text { with } \mathfrak{u}(0) \leq \mathfrak{v}(0),
$$

then for all $t>0$ we have $\mathfrak{u}(t) \leq \mathfrak{v}(t)$.

Proof. First, we easily see that for all $t>0$ we have

$$
\frac{\mathrm{d}}{\mathrm{d} t}\left(e^{t} \mathfrak{u}(t)\right) \leq e^{t} \mathcal{F}(\mathfrak{u}(t)), \text { and } \frac{\mathrm{d}}{\mathrm{d} t}\left(e^{t} \mathfrak{v}(t)\right) \geq e^{t} \mathcal{F}(\mathfrak{v}(t))
$$

such that

$$
\begin{aligned}
\mathfrak{u}(t)-\mathfrak{v}(t) & \leq e^{-t}(\mathfrak{u}(0)-\mathfrak{v}(0))+\int_{0}^{t} e^{-(t-\tau)} \mathcal{K} *_{d}(S(\mathfrak{u}(\tau))-S(\mathfrak{v}(\tau))) \mathrm{d} \tau \\
& \leq s_{m} \int_{0}^{t} e^{-(t-\tau)} \mathcal{K} *_{d}[\mathfrak{u}(\tau)-\mathfrak{v}(\tau)]_{+} \mathrm{d} \tau
\end{aligned}
$$

where $[\mathfrak{u}(t)-\mathfrak{v}(t)]_{+}=\left(\left[u_{n}(t)-v_{n}(t)\right]_{+}\right)_{n \in \mathbb{Z}}$ and $[\cdot]_{+}$stands for the positive part. We can then define

$$
\rho_{\lambda}(t):=\sup _{n \in \mathbb{Z}}\left[u_{n}(t)-v_{n}(t)\right]_{+} e^{-\lambda t},
$$

and $\bar{\rho}_{\lambda}:=\sup _{t \in[0, T]} \rho_{\lambda}(t)$ for some arbitrary $T>0$ and $\lambda$ that will be fixed later. We readily obtain that

$$
\rho_{\lambda}(t) \leq s_{m} \bar{\rho}_{\lambda} \int_{0}^{T} e^{-(1+\lambda) \tau} \mathrm{d} \tau
$$

and thus

$$
\bar{\rho}_{\lambda}\left(1-\frac{s_{m}}{1+\lambda}\right) \geq \bar{\rho}_{\lambda}\left(1-s_{m} \int_{0}^{T} e^{-(1+\lambda) \tau} \mathrm{d} \tau\right) \geq 0 .
$$

Then, there exists $\lambda_{*}>0$ large enough such that for all $\lambda \geq \lambda_{*}$ we have $\bar{\rho}_{\lambda} \leq 0$. As a conclusion, we have shown that for any $T>0, \mathfrak{u}(t) \leq \mathfrak{v}(t)$ for all $t \in[0, T]$, which concludes the proof. 
Now, we are going to extend a result from [3] to our discrete setting. Here we assume that $\tau_{0}>\tau$, $c \neq 0, \mathbf{R}_{1} \subset \mathbb{R}$ is an open set and $\mathbf{R}_{2}=\mathbb{R} \backslash \mathbf{R}_{1},(x, t) \mapsto g(x, t) \in L^{\infty}\left(\mathbb{R} \times\left[\tau, \tau_{0}\right]\right)$ and the sequence of functions $\left((x, t) \mapsto \mathbf{K}_{j}(x, t)\right)_{j \in \mathbb{Z}}$ is such that there exists $k_{0}>0, k_{1}$ so that for any $(x, t) \in \mathbb{R} \times\left[\tau, \tau_{0}\right]$ the sequence $\left(\mathbf{K}_{j}(x, t)\right)_{j \in \mathbb{Z}}$ satisfies

$$
k_{0} K_{j} \leq \mathbf{K}_{j}(x, t) \leq k_{1} K_{j} \text { for all } j \in \mathbb{Z}
$$

where $\left(K_{j}\right)_{j \in \mathbb{Z}}$ a sequence satisfying Hypothesis (H2). We will denote $\mathbf{K} \cdot \mathbf{u}(x, t):=\sum_{j \in \mathbb{Z}} \mathbf{K}_{j}(x, t) \mathbf{u}(x-$ $j, t)$ for all $(x, t) \in \mathbb{R} \times\left[\tau, \tau_{0}\right]$.

Lemma A.2. Assume that $\mathbf{u} \in \mathscr{C}^{1}\left(\left[\tau, \tau_{0}\right], L^{\infty}(\mathbb{R})\right) \cap \mathscr{C}\left(\left[\tau, \tau_{0}\right], W^{1, \infty}(\mathbb{R})\right)$ and suppose that $\mathbf{u}(x, t) \geq$ 0 for all $t \in\left[\tau, \tau_{0}\right]$ and $x \in \mathbf{R}_{2}$ and $\mathbf{u}$ satisfies

$$
\partial_{t} \mathbf{u}-c \partial_{x} \mathbf{u}-g(x, t) \mathbf{u}-\mathbf{K} \cdot \mathbf{u}(x, t) \geq 0
$$

$t \in\left[\tau, \tau_{0}\right]$ and $x \in \mathbf{R}_{1}$. If $u(x, \tau) \geq 0$ for all $x \in \mathbb{R}$, then $\mathbf{u}(x, t) \geq 0$ for all $t \in\left[\tau, \tau_{0}\right]$ and $x \in \mathbb{R}$. Moreover, if $\mathbf{u}(\cdot, \tau) \neq 0$ on $\mathbb{R}$ then $\mathbf{u}(x, t)>0$ for all $t \in\left[\tau, \tau_{0}\right]$ and $x \in \mathbf{R}_{1}$.

Proof. Without loss of generality we may assume that $\tau=0$. Up to slight modification of the subset $\mathbb{R}_{1}$ and $\mathbb{R}_{2}$, as outlined in [3], we can also consider the case where $c=0$ in (A.2). By our assumption on the regularity of $\mathbf{u}$, if the first conclusion of the Lemma is not true, then there exists $\epsilon>0$ and $T \in\left(0, \tau_{0}\right]$ such that $\mathbf{u}(x, t)>-\epsilon e^{2 \kappa t}$ for all $0<t<T$ and $x \in \mathbb{R}$ with $\inf _{x \in \mathbb{R}} \mathbf{u}(x, t)=-\epsilon e^{2 \kappa T}$ where $\kappa=\sup _{(x, t) \in \mathbb{R} \times\left[\tau, \tau_{0}\right]}|g(x, t)|+k_{1}$. Therefore, there exists an interval $[a, b]$ such that $\mathbf{u}(x, T) \leq-\frac{15}{16} \epsilon e^{2 \kappa T}$ for $x \in[a, b]$. Following the strategy of [3], we introduce a smooth function $\zeta(x)$ such that $\zeta(x)=\min _{x \in \mathbb{R}} \zeta(x)=1$ for $x \in[a, b], \sup _{x \in \mathbb{R}} \zeta(x)=\zeta( \pm \infty)=3$ and $\left|\zeta^{\prime}(x)\right| \leq 1$. We definte $\mathbf{w}_{\sigma}(x, t)=-\epsilon\left(\frac{3}{4}+\sigma \zeta(x)\right) e^{2 \kappa t}$ for $\sigma \in[0,1]$. As by construction we have $\mathbf{w}_{1 / 4}(x, t) \leq \mathbf{u}(x, t)$ for all $t \in[0, T]$ and $x \in \mathbb{R}$ and $\mathbf{w}_{1 / 8}(x, T)>\mathbf{u}(x, T)$ for $x \in[a, b]$ there exists a minimum $\sigma_{*} \in(1 / 8,1 / 4]$ such that $\mathbf{w}_{\sigma_{*}}(x, t) \leq \mathbf{u}(x, t)$ for $t \in[0, T]$ and $x \in \mathbb{R}$. We claim that there exist a sequence $\left(x_{n}, t_{n}\right) \in \mathbf{R}_{1}$ and $\left(x_{0}, t_{0}\right)$ such that inequality (A.2) respectively holds for (i) $\left(x_{n}, t_{n}\right)$; (ii) $\lim _{n \rightarrow \infty}\left(x_{n}, t_{n}\right)=\left(x_{0}, t_{0}\right)$; (iii) $\lim _{n \rightarrow \infty}\left(\mathbf{u}\left(x_{n}, t_{n}\right)-\mathbf{w}_{\sigma_{*}}\left(x_{n}, t_{n}\right)\right)=0$ the infimum of $\mathbf{u}(x, t)-\mathbf{w}_{\sigma_{*}}(x, t)$ on $\mathbb{R} \times[0, T]$. The proof of the claim can be found in [3] and is omitted. As a consequence, we have

$$
\begin{aligned}
0 & \geq \lim _{n \rightarrow \infty} \partial_{t}\left(\mathbf{u}-\mathbf{w}_{\sigma_{*}}\right)\left(x_{n}, t_{n}\right) \\
& \geq \liminf _{n \rightarrow \infty}(\mathbf{K} \cdot \mathbf{u})\left(x_{n}, t_{n}\right)-2 \kappa \mathbf{w}_{\sigma_{*}}\left(x_{0}, t_{0}\right)+\sup _{(x, t) \in \mathbb{R} \times\left[\tau, \tau_{0}\right]}|g(x, t)| \mathbf{w}_{\sigma_{*}}\left(x_{0}, t_{0}\right) \\
& \geq\left(k_{1}+\sup _{(x, t) \in \mathbb{R} \times\left[\tau, \tau_{0}\right]}|g(x, t)|-2 \kappa\right) \mathbf{w}_{\sigma_{*}}\left(x_{0}, t_{0}\right)>0,
\end{aligned}
$$

which is a contradiction, therefore $\mathbf{u}(x, t) \geq 0$ for all $x \in \mathbb{R}$ and $t \in\left[\tau, \tau_{0}\right]$.

For the last part of the conclusion, we define $\mathbf{v}(x, t)=e^{\kappa t} \mathbf{u}(x, t)$ then we have $\partial_{t} \mathbf{v}(x, t) \geq k_{0} \mathcal{K}_{\delta} *$ $\mathbf{v}(x, t)=k_{0} \sum_{j \in \mathbb{Z}} K_{j} \mathbf{v}(x-j, t)$ for $x \in \mathbf{R}_{1}$ and $t \in\left[\tau, \tau_{0}\right]$ since $\mathbf{u}(x, t) \geq 0$. Then we have 
$\mathbf{v}(x, t) \geq t k_{0} \mathcal{K}_{\delta} * \mathbf{v}(x, 0)$ for $0 \leq t$ and after $N$ iterations we get

$$
\mathbf{v}(x, t) \geq k_{0} \frac{t^{N}}{N !} \mathcal{K}_{\delta} * \cdots * \mathcal{K}_{\delta} * \mathbf{v}(x, 0), \quad x \in \mathbf{R}_{1} .
$$

As we have $K_{ \pm 1}>0$ and that $\mathbf{u}(\cdot, \tau) \neq 0$ on $\mathbb{R}$ there always exists $N$ large enough such that $\mathcal{K}_{\delta} * \cdots * \mathcal{K}_{\delta} * \mathbf{v}(x, 0)>0$ and thus $\mathbf{u}(x, t)>0$ on $\mathbf{R}_{1}$.

\section{References}

[1] N.D. Alikakos, P.W. Bates and X. Chen. Periodic traveling waves and locating oscillating patterns in multidimensional domains. Trans. of the AMS, vol 351, no 7, (1999), pp. 27772805 .

[2] S.-I. Amari. Dynamics of pattern formation in lateral-inhibition type neural fields. Biological Cybernetics, 27(2), (1977), pp. 77-87.

[3] P. W. Bates and F. Chen. Spectral analysis of traveling waves for nonlocal evolution equations. SIAM J. Math. Anal., vol 38, no 1, (2006), pp. 116-126.

[4] P. W. Bates and A. Chmaj. A Discrete Convolution Model for Phase Transitions. Arch. Rational Mech. Anal. 150 (1999) pp. 281-305.

[5] P. W. Bates, X. Chen and A. Chmaj. Traveling waves of bistable dynamics on a lattice. SIAM J. Math. Anal. vol 35, no. 2, (2003) pp. 520-546.

[6] S. Benzoni-Gavage, P. Huot and F. Rousset. Nonlinear stability analysis of semidiscrete shock waves. SIAM J. Math. Anal. vol 35, no. 3, (2003) pp. 639-707.

[7] P.C. Bressloff. Waves in Neural Media: From single Neurons to Neural Fields. Lecture notes on mathematical modeling in the life sciences, Springer, (2014).

[8] P.C. Bressloff. Spatiotemporal Dynamics of Continuum Neural Fields. J. Phys. A: Math. Theor., vol 45, 109pp, (2012).

[9] J.M. Bos. Fredholm eigenschappen van systemen met interactie over een oneindig bereik. Bachelor thesis, Leiden University, (2015).

[10] X. Chen. Existence, Uniqueness, and asymptotic stability of traveling waves in nonlocal evolution equations. Adv. Diff. Eq., 2 (1997), pp. 125-160.

[11] S. Coombes. Waves, bumps, and patterns in neural fields theories. Biological Cybernetics, vol. 93, no. 2, (2005) pp. 91-108.

[12] G.B. Ermentrout. Neural networks as spatio-temporal pattern-forming systems. Reports on Progress in Physics, 61, (1998), pp. 353-430. 
[13] G. B. Ermentrout and J. B. McLeod. Existence and uniqueness of travelling waves for a neural network. Proceedings of the Royal Society of Edimburghn 123A (1993) pp. 461-478.

[14] J. Fang and G. Faye. Monotone traveling waves for delayed neural field equations. Mathematical Methods and Models in Applied Sciences, vol 26, no 10 (2016), pp. 1919-1954.

[15] G. Faye. Existence and stability of traveling pulses of a neural field equation with synaptic depression. SIAM J. Appl. Dyn. Syst, 12-4, (2013) pp. 2032-2067.

[16] G. Faye and A. Scheel. Fredholm properties of nonlocal differential equations via spectral flow. Indiana Univ. Math. J., 63 (2014), pp. 1311-1348.

[17] G. Faye and A. Scheel Existence of pulses in excitable media with nonlocal coupling. Advances in Mathematics, vol 270 (2015), pp. 400-456.

[18] A. Hoffman and M. Holzer. Invasion fronts on graphs: the Fisher-KPP equation on homogeneous trees and Erdös-Réyni graphs. Submitted, (2016), pp. 1-26.

[19] J.J. Hopfield. Neurons with graded response have collective computational properties like those of two-state neurons. Proc. Natl. Acad. Sci. USA, vol 81, (1984) pp. 3088-3092.

[20] H.J. Hupkes and B. Sandstede. Stability of Pulse Solutions for the Discrete FitzHugh-Nagumo System. Transactions of the AMS 365, (2013), pp. 251-301.

[21] N. E. Kouvaris, H. Kori, and A. S. Mikhailov. Traveling and pinned fronts in bistable reactiondiffusion systems on networks. PLoS ONE, 7(9): (2012) pp.1-12.

[22] J. Mallet-Paret. The Fredholm Alternative for Functional Differential Equations of Mixed Type. Journal of Dynamics and Differential Equations, 11, 1, (1999), pp. 1-47.

[23] J. Mallet-Paret. The global structure of traveling waves in spatially discrete dynamical systems. Journal of Dynamics and Differential Equations, 11, 1, (1999), pp. 49-127.

[24] T.K. Sato, I. Nauhaus and M. Carandini. Traveling waves in visual cortex. Neuron Review, $75,(2012)$, pages $218-229$.

[25] W. Schouten and H.J. Hupkes. Nonlinear stability of pulse solutions for the discrete FitzHughNagumo equation with infinite-range interactions. Submitted, (2017) pp. 1-92.

[26] H.R. Wilson and J.D. Cowan. A mathematical theory of the functional dynamics of cortical and thalamic nervous tissue. Biological Cybernetics, 13(2) (1973), pp. 55-80. 